\title{
GRAPH THEORETIC STRUCTURE OF MAPS OF THE CANTOR SPACE
}

\author{
NILSON C. BERNARDES JR. AND UDAYAN B. DARJI
}

\begin{abstract}
In this paper we develop unifying graph theoretic techniques to study the dynamics and the structure of spaces $\mathcal{H}\left(\{0,1\}^{\mathbb{N}}\right)$ and $\mathcal{C}\left(\{0,1\}^{\mathbb{N}}\right)$, the space of homeomorphisms and the space of self-maps of the Cantor space, respectively. Using our methods, we give characterizations which determine when two homeomorphisms of the Cantor space are conjugate to each other. We also give a new characterization of the comeager conjugacy class of the space $\mathcal{H}\left(\{0,1\}^{\mathbb{N}}\right)$. The existence of this class was established by Kechris and Rosendal and a specific element of this class was described concretely by Akin, Glasner and Weiss. Our characterization readily implies many old and new dynamical properties of elements of this class. For example, we show that no element of this class has a Li-Yorke pair, implying the well known GlasnerWeiss result that there is a comeager subset of $\mathcal{H}\left(\{0,1\}^{\mathbb{N}}\right)$ each element of which has topological entropy zero. Our analogous investigation in $\mathcal{C}\left(\{0,1\}^{\mathbb{N}}\right)$ yields a surprising result: there is a comeager subset of $\mathcal{C}\left(\{0,1\}^{\mathbb{N}}\right)$ such that any two elements of this set are conjugate to each other by an element of $\mathcal{H}\left(\{0,1\}^{\mathbb{N}}\right)$. Our description of this class also yields many old and new results concerning dynamics of a comeager subset of $\mathcal{C}\left(\{0,1\}^{\mathbb{N}}\right)$.
\end{abstract}

\section{INTRODUCTION}

In recent years $\mathcal{H}\left(\{0,1\}^{\mathbb{N}}\right)$, the group of homeomorphisms of the Cantor space, has enjoyed attention from mathematicians working in diverse fields such as dynamical systems 4, 16 and model theory 18. From the dynamics point of view, $\{0,1\}^{\mathbb{N}}$ is the symbol space on two variables and a fundamental tool in analyzing topological dynamics of complicated systems. From the model theory point of view, $\mathcal{H}\left(\{0,1\}^{\mathbb{N}}\right)$ is important as it is isomorphic to the group of automorphisms of the countable, atomless boolean algebra. The study of $\mathcal{H}\left(\{0,1\}^{\mathbb{N}}\right)$ from the dynamical systems approach utilizes analytic techniques while the model theory viewpoint exploits fact that $\{0,1\}^{\mathbb{N}}$ can be viewed as the Fraïssè limit of finite Boolean algebras. Our approach is fundamentally different in that to each $h \in \mathcal{H}\left(\{0,1\}^{\mathbb{N}}\right)$ and each finite partition $\mathcal{P}$ of clopen subsets of $\{0,1\}^{\mathbb{N}}$, we associate a digraph $\operatorname{Gr}(h, \mathcal{P})$. We use geometric and graph theoretic properties of these digraphs to deduce dynamical properties of $h$.

In one of our main results, we establish characterizations of when two elements of $\mathcal{H}\left(\{0,1\}^{\mathbb{N}}\right)$ are topologically conjugate to each other. In addition to its importance from the algebraic point of view, this result is also very important from the dynamical systems point of view. The reason for this is that two topologically conjugate homeomorphisms have the same topological dynamics. Hence, dynamical

2000 Mathematics Subject Classification. Primary: 37B99, 54H20, Secondary: 22D05, 05C20.

Key words and phrases. Cantor Space; Homeomorphisms; Chaos; Conjugacy relation. 
properties of every element of a conjugacy class can be described by the dynamical properties of a single member of that class. Characterizations of the conjugacy relation for other groups such as the symmetric group on $\mathbb{N}$, the group of automorphisms of the random graph and the group of order preserving automorphisms of the rationals were described by Truss in 20 .

Truss [20] also defined the concept of generics in a Polish group. We say that a Polish group $G$ admits generics if $G$ has a comeager conjugacy class. In this case, we say that a generic element of $G$ has Property $P$ if every element of the comeager conjugacy class has Property P. We caution the reader that in dynamical systems, topology and other fields, the word generic is often used in a different manner. Namely, it refers to a comeager subset of a complete metric space with no reference to algebraic structure. We will use the phrase "comeager conjugacy class" instead of the word "generic" to avoid confusion.

In 2001 Glasner and Weiss [15] showed that $\mathcal{H}\left(\{0,1\}^{\mathbb{N}}\right)$ has a dense conjugacy class. They called the property of having a dense conjugacy class the topological Rohlin property. This was also shown by Akin, Hurley and Kennedy in their monograph [4. In the same monograph they raised the question whether $\mathcal{H}\left(\{0,1\}^{\mathbb{N}}\right)$ has a comeager conjugacy class. This was settled affirmatively in 2007 by Kechris and Rosendal in [18] using model theoretic techniques. Akin, Glasner and Weiss [3], in 2008, gave a concrete construction of what they called a "Special Homeomorphism" whose conjugacy class is comeager. As an application of the techniques developed in the present article, we give a geometric/graph theoretic description of the elements of this comeager conjugacy class. We describe these homeomorphisms below to give a flavor of the ideas developed here.

Let $h \in \mathcal{H}\left(\{0,1\}^{\mathbb{N}}\right)$ and $\mathcal{P}$ be a finite partition of clopen subsets of $\{0,1\}^{\mathbb{N}}$. Then, $\operatorname{Gr}(h, \mathcal{P})$ is a digraph whose vertex set is $\mathcal{P}$ and $\overrightarrow{a b}$ is an edge of $\operatorname{Gr}(h, \mathcal{P})$ if and only if $h(a) \cap b \neq \emptyset$. A digraph $H$ is a loop if the vertex set of $H$ is $\left\{v_{1}, \ldots, v_{n}\right\}$ and the edges of $H$ are $\overrightarrow{v_{n} v_{1}}$ and $\overrightarrow{v_{i} v_{i+1}}$ for $1 \leq i<n$. In this case, we say that $H$ is a loop of length $n$. A digraph $H$ is a dumbbell if the vertex set of $H$ is the union of three disjoint sets $l_{1}=\left\{u_{1}, \ldots, u_{r}\right\}, p=\left\{v_{1}, \ldots, v_{s}\right\}, l_{2}=\left\{w_{1}, \ldots, w_{t}\right\}$ and the edges of $H$ are

- the edges of the loops formed by $l_{1}$ and $l_{2}$,

- the edges of the path $p$, i.e., $\overrightarrow{v_{i} v_{i+1}}$ for $1 \leq i<s$, and

- $\overrightarrow{u_{1} v_{1}}, \overrightarrow{v_{s} w_{1}}$.

In this case we say that $H$ is a dumbbell of type $(r, s, t)$. If $r=t$, then we say that the dumbbell is balanced with plate weight $r$. Assume that a component $H$ of $\operatorname{Gr}(h, \mathcal{P})$ is a dumbbell and let us denote $H$ as above. We say that $H$ contains a left loop of $h$ (resp. a right loop of $h$ ) if there is a nonempty clopen subset $a$ of $u_{1}$ (resp. of $w_{1}$ ) such that $h^{r}(a)=a$ (resp. $\left.h^{t}(a)=a\right)$.

Now we are ready to describe the comeager conjugacy class of $\mathcal{H}\left(\{0,1\}^{\mathbb{N}}\right)$.

Theorem. The set of all $h \in \mathcal{H}\left(\{0,1\}^{\mathbb{N}}\right)$ with the following property is a comeager conjugacy class of $\mathcal{H}\left(\{0,1\}^{\mathbb{N}}\right)$ :

For every $m \in \mathbb{N}$, there are a partition $\mathcal{P}$ of $\{0,1\}^{\mathbb{N}}$ of mesh $<1 / m$ and a multiple $q \in \mathbb{N}$ of $m$ such that every component of $\operatorname{Gr}(h, \mathcal{P})$ is a balanced dumbbell with plate weight $q$ ! that contains both a left and a right loop of $h$. 
We point out that using projective Fraïssè limits, Kwiatkowska 17 has shown that $\mathcal{H}\left(\{0,1\}^{\mathbb{N}}\right)$ has ample generics, a property stronger than having a comeager conjugacy class. We would also like to point out that the ideas used by Akin, Hurley and Kennedy in 4 to prove that $\mathcal{H}\left(\{0,1\}^{\mathbb{N}}\right)$ has a dense conjugacy class has some distant resemblance to our techniques.

Using our graph theoretic techniques, we prove a surprising result: there is a comeager subset of $\mathcal{C}\left(\{0,1\}^{\mathbb{N}}\right)$ such that any two elements of this set are conjugate to each other by an element of $\mathcal{H}\left(\{0,1\}^{\mathbb{N}}\right)$. This is done by giving a geometric/graph theoretic description of this class in a manner similar to that of $\mathcal{H}\left(\{0,1\}^{\mathbb{N}}\right)$.

The notion of chaos is another well studied concept in topological dynamics. There are several different notions of chaos. For instance, page 1306 of 2 contains a table with 11 notions of chaos and the relationships among them, including the 4 best known notions, namely: positive topological entropy, chaos in the sense of Devaney, weak mixing and chaos in the sense of $\mathrm{Li}$ and Yorke. As is well-known, Li-Yorke chaos is the weakest of all notions of chaos [7. In 15] Glasner and Weiss showed that a comeager subset of $\mathcal{H}\left(\{0,1\}^{\mathbb{N}}\right)$ has topological entropy zero. Hence in some sense an element chosen at "random" from $\mathcal{H}\left(\{0,1\}^{\mathbb{N}}\right)$ has topological entropy zero and therefore is not chaotic in this sense. As a simple corollary to our investigation, we show that homeomorphisms of the comeager conjugacy class have a much stronger property, namely that they have no Li-Yorke pair.

We show that homeomorphisms of this comeager conjugacy class also have other properties which make them tame. In this direction we show that each such homeomorphism has the shadowing property and the restriction of the homeomorphism to each of its $\omega$-limit sets is topologically conjugate to the universal odometer. Hochman [16] showed that among all the transitive homeomorphisms of the Cantor space, the set of homeomorphisms topologically conjugate to the universal odometer is comeager. We also show that for all $h$ in the comeager conjugacy class, the set of recurrent points of $h$ is equal to the set of chain recurrent points of $h$. Moreover, $h$ is chain continuous on a dense open subset of $\{0,1\}^{\mathbb{N}}$ but not equicontinuous on an uncountable set.

We also show analogous dynamical properties of a comeager subset of $\mathcal{C}\left(\{0,1\}^{\mathbb{N}}\right)$. In particular, we show that the set of all $f \in \mathcal{C}\left(\{0,1\}^{\mathbb{N}}\right)$ which have the following properties is comeager in $\mathcal{C}\left(\{0,1\}^{\mathbb{N}}\right)$ :

- $f$ has no Li-Yorke pair.

- The restriction of $f$ to each of its $\omega$-limit sets is topologically conjugate to the universal odometer.

- $f$ is chain continuous at every point.

Earlier it was shown in [13] that the set of $f \in \mathcal{C}\left(\{0,1\}^{\mathbb{N}}\right)$ with topological entropy zero and no periodic point is comeager in $\mathcal{C}\left(\{0,1\}^{\mathbb{N}}\right)$. In [14] it was shown that there is a comeager set of $f \in \mathcal{C}\left(\{0,1\}^{\mathbb{N}}\right)$ such that for a comeager set of $\sigma \in\{0,1\}^{\mathbb{N}}$, the restriction of $f$ to the $\omega$-limit set of $f$ at $\sigma$ is topologically conjugate to the universal odometer.

This paper is organized as follows: Section 2 develops properties of $\operatorname{Gr}(f, \mathcal{P})$ for $f \in \mathcal{C}\left(\{0,1\}^{\mathbb{N}}\right)$ and for $f \in \mathcal{H}\left(\{0,1\}^{\mathbb{N}}\right)$, and contains an important approximation theorem, Theorem 2.5 In Section 3 we give characterizations of when two homeomorphisms (or two continuous maps) of the Cantor space are topologically conjugate to each other. Section 4 contains an useful geometric/graph theoretic 
description of the homeomorphisms of the Cantor space whose conjugacy class is comeager. Moreover, by applying this description we obtain several results concerning dynamical properties of the comeager conjugacy class of $\mathcal{H}\left(\{0,1\}^{\mathbb{N}}\right)$. In Section 5 we prove the surprising result that there is a comeager subset of $\mathcal{C}\left(\{0,1\}^{\mathbb{N}}\right)$ such that any two elements of this set are conjugate to each other by an element of $\mathcal{H}\left(\{0,1\}^{\mathbb{N}}\right)$. Using the description of this set, we prove dynamical properties of elements of this set.

\section{Approximation Theorem}

By Cantor space we mean any compact, 0-dimensional metric space without isolated points. The principal model of the Cantor space we use is $\{0,1\}^{\mathbb{N}}$ endowed with the product topology, where $\{0,1\}$ is given the discrete topology. This topology is generated by the metric $d(\sigma, \tau)=\frac{1}{n}$ where $n$ is the least positive integer where $\sigma(n) \neq \tau(n)$ if such an integer exists and $d(\sigma, \tau)=0$, otherwise.

By a partition of $\{0,1\}^{\mathbb{N}}$ we mean a finite collection of nonempty pairwise disjoint clopen sets whose union is $\{0,1\}^{\mathbb{N}}$. A map $\nu: \mathcal{P} \rightarrow \mathcal{Q}$, between partitions $\mathcal{P}$ and $\mathcal{Q}$ of $\{0,1\}^{\mathbb{N}}$, is called a refinement map if

$$
\nu(a) \supset a \text { for all } a \in \mathcal{P} .
$$

In this case, we say that $\mathcal{P}$ is a refinement of $\mathcal{Q}$. Note that a refinement map is necessarily surjective.

For each finite collection $\mathcal{C}$ of nonempty subsets of $\{0,1\}^{\mathbb{N}}$, we define the mesh of $\mathcal{C}$ by

$$
\operatorname{mesh}(\mathcal{C})=\max _{A \in \mathcal{C}} \operatorname{diam}(A)
$$

If $\sigma$ is a finite string of 0 's and 1's, then $[\sigma]$ denotes the set of all points of $\{0,1\}^{\mathbb{N}}$ which are extensions of $\sigma$. For each $n \in \mathbb{N}$, we consider the partition

$$
\mathcal{B}_{n}=\left\{[\sigma]: \sigma \in\{0,1\}^{n}\right\}
$$

of $\{0,1\}^{\mathbb{N}}$. Note that

$$
\operatorname{mesh}\left(\mathcal{B}_{n}\right) \rightarrow 0 \quad \text { as } n \rightarrow \infty
$$

Moreover, $\cup \mathcal{B}_{n}$ is a basis of clopen sets for the topology of the Cantor space.

We use $\mathcal{C}\left(\{0,1\}^{\mathbb{N}}\right)\left(\right.$ resp. $\left.\mathcal{H}\left(\{0,1\}^{\mathbb{N}}\right)\right)$ to denote the space of all continuous maps (resp. of all homeomorphisms) of the Cantor space, endowed with the following metric:

$$
\tilde{d}(f, g)=\max _{\sigma \in\{0,1\}^{\mathbb{N}}} d(f(\sigma), g(\sigma)) .
$$

If $f, g \in \mathcal{C}\left(\{0,1\}^{\mathbb{N}}\right)$ and $\mathcal{P}$ is a partition of $\{0,1\}^{\mathbb{N}}$, then $f \sim_{\mathcal{P}} g$ means that $f(\sigma)$ and $g(\sigma)$ lie in the same member of $\mathcal{P}$ for every $\sigma \in\{0,1\}^{\mathbb{N}}$. Note that

$$
f \sim_{\mathcal{P}} g \Longrightarrow \tilde{d}(f, g) \leq \operatorname{mesh}(\mathcal{P}) .
$$

Central to our investigation is the notion of a digraph (= directed graph). A digraph $G$ consists of a finite set $V(G)$ of vertices together with a set $E(G)$ of directed edges between vertices. By a left end of $G$ (resp. a right end of $G$ ) we mean a vertex $v$ of $G$ that has no incoming edge (resp. no outgoing edge). We say that $G$ is a digraph without right ends (resp. a digraph without ends) if $G$ has no right end (resp. no left end and no right end). If $G$ and $H$ are digraphs, then a digraph map $\phi: H \rightarrow G$ is a map from the vertex set of $H$ into the vertex set of 
$G$ such that $\overrightarrow{\phi(u) \phi(v)}$ is an edge of $G$ whenever $\overrightarrow{u v}$ is an edge of $H$. We say that $\phi$ is surjective when it is a surjection between the sets of vertices (but it need not be surjective on the sets of edges). By a component of a digraph $G$ we simply mean a largest (in vertices and edges) subgraph $H$ of $G$ such that given any two vertices $a, b$ in $H$, there are vertices $a_{1}, \ldots, a_{n}$ in $H$ such that $a_{1}=a, a_{n}=b$ and for any $1 \leq i<n, \overrightarrow{a_{i} a_{i+1}}$ or $\overrightarrow{a_{i+1} a_{i}}$ is an edge of $H$.

To each $f \in \mathcal{C}\left(\{0,1\}^{\mathbb{N}}\right)$ and each partition $\mathcal{P}$ of $\{0,1\}^{\mathbb{N}}$, we associate a digraph $\operatorname{Gr}(f, \mathcal{P})$ in the following fashion: the vertices of $\operatorname{Gr}(f, \mathcal{P})$ are the elements of $\mathcal{P}$ and for sets $a, b \in \mathcal{P}$, directed edge $\overrightarrow{a b} \in \operatorname{Gr}(f, \mathcal{P})$ if and only if $f(a) \cap b \neq \emptyset$. Note that $\operatorname{Gr}(f, \mathcal{P})$ is always a digraph without right ends. If $f$ is surjective, then $\operatorname{Gr}(f, \mathcal{P})$ is a digraph without ends. If $\nu: \mathcal{P} \rightarrow \mathcal{Q}$ is a refinement map, then $\nu: \operatorname{Gr}(f, \mathcal{P}) \rightarrow \operatorname{Gr}(f, \mathcal{Q})$ is a surjective graph map (in the present case, it is surjective on edges too). Technically speaking,

$$
\mathcal{P} \rightarrow \operatorname{Gr}(f, \mathcal{P})
$$

is a functor from the category of partitions of the Cantor space (whose morphisms are the refinement maps) to the category of digraphs (whose morphisms are the digraph maps). Moreover,

$$
f \sim_{\mathcal{P}} g \Longrightarrow \operatorname{Gr}(f, \mathcal{P})=\operatorname{Gr}(g, \mathcal{P}) .
$$

Theorem 2.1. Let $G$ be a digraph without right ends whose vertex set $\mathcal{P}$ is a partition of $\{0,1\}^{\mathbb{N}}$ and define

$$
X=\bigcup\{a \in \mathcal{P}: a \text { is a left end of } G\} .
$$

Then, there is a homeomorphism $f$ from $\{0,1\}^{\mathbb{N}}$ onto $\{0,1\}^{\mathbb{N}} \backslash X$ such that

$$
\operatorname{Gr}(f, \mathcal{P})=G \text {. }
$$

In particular, if $G$ is a digraph without ends, then $f \in \mathcal{H}\left(\{0,1\}^{\mathbb{N}}\right)$.

Proof. For each $a \in \mathcal{P}$, let $O_{a}$ be a partition of $a$ whose cardinality is equal to the number of edges of $G$ going out $a$. For each $b \in \mathcal{P}$ which is not a left end of $G$, let $I_{b}$ be a partition of $b$ whose cardinality is equal to the number of edges of $G$ coming into $b$. For each edge $e=\overrightarrow{a b} \in G$, we define an ordered pair $\left(x_{e}, y_{e}\right)$ such that

- $x_{e} \in O_{a}, y_{e} \in I_{b}$ and

- if $e$ and $e^{\prime}$ are two distinct edges in $G$, then $x_{e} \neq x_{e^{\prime}}$ and $y_{e} \neq y_{e^{\prime}}$.

We note that the second condition above implies that $x_{e} \cap x_{e^{\prime}}=\emptyset$ and $y_{e} \cap y_{e^{\prime}}=\emptyset$. Now, let $f$ be a homeomorphism from $\{0,1\}^{\mathbb{N}}$ onto $\{0,1\}^{\mathbb{N}} \backslash X$ such that

$$
f\left(x_{e}\right)=y_{e} \quad \text { for each edge } e \in G \text {. }
$$

Then, $\operatorname{Gr}(f, \mathcal{P})=G$.

Theorem 2.2. Let $f \in \mathcal{C}\left(\{0,1\}^{\mathbb{N}}\right)$ and $\mathcal{Q}$ be a partition of $\{0,1\}^{\mathbb{N}}$. Assume that $G$ is a digraph without right ends and that $\phi: G \rightarrow \operatorname{Gr}(f, \mathcal{Q})$ is a surjective graph map. Then:

(a) There exists a refinement map $\nu: \mathcal{P} \rightarrow \mathcal{Q}$ and a bijection $\psi$ from $\mathcal{P}$ onto the vertex set of $G$ such that $\nu=\phi \circ \psi$.

(b) There exists $g \in \mathcal{C}\left(\{0,1\}^{\mathbb{N}}\right)$ such that $\psi: \operatorname{Gr}(g, \mathcal{P}) \rightarrow G$ is a digraph isomorphism. Moreover, if $G$ is a digraph without ends, then we may take such a $g$ in $\mathcal{H}\left(\{0,1\}^{\mathbb{N}}\right)$. 
(c) For any $g$ as in (b),

$$
\begin{aligned}
& \tilde{d}(f, g) \leq \operatorname{mesh}(\mathcal{Q})+\operatorname{mesh}(f(\mathcal{Q})), \\
& \text { where } f(\mathcal{Q})=\{f(a): a \in \mathcal{Q}\} .
\end{aligned}
$$

Proof. (a) For each $a \in \mathcal{Q}$, choose a partition $\mathcal{P}_{a}$ of $a$ with the same cardinality as $\phi^{-1}(a)$. Consider the partition

$$
\mathcal{P}=\bigcup_{a \in \mathcal{Q}} \mathcal{P}_{a}
$$

of $\{0,1\}^{\mathbb{N}}$ and define $\psi$ as a bijection from $\mathcal{P}_{a}$ onto $\phi^{-1}(a)$ for each $a \in \mathcal{Q}$. Then $\psi$ is a bijection from $\mathcal{P}$ onto the vertex set of $G$ and $\phi \circ \psi: \mathcal{P} \rightarrow \mathcal{Q}$ is a refinement map.

(b) There exists a unique digraph $H$ whose vertex set is $\mathcal{P}$ for which $\psi: H \rightarrow G$ is a digraph isomorphism. By Theorem 2.1, there exists $g \in \mathcal{C}\left(\{0,1\}^{\mathbb{N}}\right)$ such that

$$
\operatorname{Gr}(g, \mathcal{P})=H .
$$

Moreover, Theorem 2.1 gives $g \in \mathcal{H}\left(\{0,1\}^{\mathbb{N}}\right)$ if $H$ (or equivalently $G$ ) is a digraph without ends.

(c) Let us fix $\sigma \in\{0,1\}^{\mathbb{N}}$. Let $a, b \in \mathcal{P}$ be such that $\sigma \in a$ and $g(\sigma) \in b$. Then $\overrightarrow{a b} \in \operatorname{Gr}(g, \mathcal{P})$, which implies that

$$
\overrightarrow{\nu(a) \nu(b)}=\overrightarrow{\phi(\psi(a)) \phi(\psi(b))} \in \operatorname{Gr}(f, \mathcal{Q}) .
$$

Hence, there exists $\tau \in \nu(a)$ such that $f(\tau) \in \nu(b)$. As $f(\tau), g(\sigma) \in \nu(b)$, we have that $d(f(\tau), g(\sigma)) \leq \operatorname{mesh}(\mathcal{Q})$. As $\sigma, \tau \in \nu(a), d(f(\sigma), f(\tau)) \leq \operatorname{mesh}(f(\mathcal{Q}))$. Consequently, $d(f(\sigma), g(\sigma)) \leq \operatorname{mesh}(\mathcal{Q})+\operatorname{mesh}(f(\mathcal{Q}))$.

A digraph $\ell$ is a loop if the vertex set of $\ell$ is $\left\{v_{1}, \ldots, v_{n}\right\}$ and the edges of $\ell$ are $\overrightarrow{v_{n} v_{1}}$ and $\overrightarrow{v_{i} v_{i+1}}$ for $1 \leq i<n$. In this case, we say that $\ell$ is a loop of length $n$.

A digraph $B$ is a balloon if the vertex set of $B$ is the union of two disjoint sets $p=\left\{v_{1}, \ldots, v_{s}\right\}$ and $\ell=\left\{w_{1}, \ldots, w_{t}\right\}$, and the edges of $B$ are

- the edges of the path $p$, i.e., $\overrightarrow{v_{i} v_{i+1}}$ for $1 \leq i<s$,

- the edges of the loop formed by $\ell$, and

- $\overrightarrow{v_{s} w_{1}}$.

In such case we say that $B$ is a balloon of type $(s, t)$ and we call $v_{1}$ the initial vertex of $B$. Whenever we write a balloon $B$ simply as

$$
B=\left\{v_{1}, \ldots, v_{s}\right\} \cup\left\{w_{1}, \ldots, w_{t}\right\},
$$

we implicitly assume that it is the balloon described above.

A digraph $D$ is a dumbbell if the vertex set of $D$ is the union of three disjoint sets $l_{1}=\left\{u_{1}, \ldots, u_{r}\right\}, p=\left\{v_{1}, \ldots, v_{s}\right\}$ and $l_{2}=\left\{w_{1}, \ldots, w_{t}\right\}$, and the edges of $D$ are

- the edges of the loops formed by $l_{1}$ and $l_{2}$,

- the edges of the path $p$, i.e., $\overrightarrow{v_{i} v_{i+1}}$ for $1 \leq i<s$, and

- $\overrightarrow{u_{1} v_{1}}, \overrightarrow{v_{s} w_{1}}$. 
In this case we say that $D$ is a dumbbell of type $(r, s, t)$. If $r=t$, then we say that the dumbbell is balanced with plate weight $r$. We say that $s$ is the length of the bar of the dumbbell. Whenever we write a dumbbell $D$ simply as

$$
D=\left\{u_{1}, \ldots, u_{r}\right\} \cup\left\{v_{1}, \ldots, v_{s}\right\} \cup\left\{w_{1}, \ldots, w_{t}\right\},
$$

we implicitly assume that it is the dumbbell described above.

Lemma 2.3. (a) Let $G$ be a digraph without right ends. For any edge e $=\overrightarrow{u v}$ of $G$, there exist positive integers $S$ and $M$ so that if $s \geq S$ and $m$ is a positive integer multiple of $M$, then a balloon of type $(s, m)$ admits a graph map into $G$ such that $u$ is the image of the initial vertex of the balloon.

(b) Let $G$ be a digraph without ends. For any edge $e=\overrightarrow{u v}$ of $G$, there exist positive integers $N, S$ and $M$ so that if $s \geq S$ and $n, m$ are positive integer multiples of $N, M$, respectively, then a dumbbell of type $(n, s, m)$ admits a graph map into $G$ such that e is the image of a bar edge in the dumbbell.

Proof. (a) Since $G$ is a digraph without right ends, we can start with edge $e$ and continue a path in $G$ to the right an arbitrary number of steps. Since there are only finitely many vertices, we obtain a pseudo-balloon in $G$, that is, a path

$$
u=u_{1}, v=u_{2}, \ldots, u_{S}, u_{S+1}, \ldots, u_{S+M}, u_{S+M+1}
$$

with

$$
u_{S+M+1}=u_{S+1} .
$$

We call it a pseudo-balloon because the vertices need not be distinct. If $s \geq S$ and $m$ is a positive integer multiple of $M$, then we can extend the bar length from $S$ to $s$ by moving into the pseudo-loop and continuing aroud it. Then we can go around the pseudo-loop as often as we want to obtain a pseudo-loop of length $m$. In this way we obtain a pseudo-balloon in $G$ of the form

$$
u=v_{1}, v=v_{2}, \ldots, v_{s}, v_{s+1}, \ldots, v_{s+m}, v_{s+m+1}=v_{s+1} .
$$

Now, let $w_{i}=\left(v_{i}, i\right)$ for $i=1, \ldots, s+m$ and consider the balloon

$$
B=\left\{w_{1}, \ldots, w_{s}\right\} \cup\left\{w_{s+1}, \ldots, w_{s+m}\right\} .
$$

The projection $w_{i} \in B \mapsto v_{i} \in G$ is the required graph map.

(b) Since $G$ is a digraph without ends, we can apply the same procedure as in (a) also to the left. In this way we obtain a pseudo-dumbbell in $G$, that is, a path

$$
u_{0}, u_{1}, \ldots, u_{N}, u_{N+1}, \ldots, u_{N+S}, u_{N+S+1}, \ldots, u_{N+S+M}, u_{N+S+M+1}
$$

with

$$
u_{N}=u_{0} \quad \text { and } \quad u_{N+S+M+1}=u_{N+S+1},
$$

so that the original edge $e=\overrightarrow{u v}$ is somewhere along the bar. Now we continue by arguing as in case (a).

Let us now establish our main graph theoretic result.

Theorem 2.4. (a) Let $G$ be a digraph without right ends. There exist positive integers $K, S$ and $M$ so that if $k \geq K, s \geq S$ and $m$ is a positive integer multiple of $M$, then a digraph consisting of $k$ disjoint balloons of type $(s, m)$ admits a graph map onto $G$.

(b) Let $G$ be a digraph without ends. There exist positive integers $K, N, S$ and $M$ so that if $k \geq K, s \geq S$ and $n, m$ are positive integer multiples of $N, M$, respectively, 
then a digraph consisting of $k$ disjoint dumbbells of type $(n, s, m)$ admits a graph map onto $G$.

Proof. We shall prove only case (b), since case (a) is analogous. Let $e_{1}, \ldots, e_{K}$ be the edges of $G$. For each $i=1, \ldots, K$, the previous lemma associates to the edge $e_{i}$ positive integers $N_{i}, S_{i}$ and $M_{i}$. Let $N$ and $M$ be the least common multiple of all the $N_{i}$ 's and of all the $M_{i}$ 's, respectively, and let $S$ be the max of the $S_{i}$ 's. If $n, m$ are multiples of $N, M$, respectively, and $s \geq S$, then the previous lemma gives us a graph map from a dumbbell $D_{i}$ of type $(n, s, m)$ into $G$ hitting $e_{i}(i=1, \ldots, K)$. Clearly, we may assume that the $D_{i}$ 's are pairwise disjoint. So, the union $H$ of the $D_{i}$ 's admits a graph map onto $G$. If we want more than $K$ dumbbells, then it is enough to get as many disjoint copies of $D_{1}$ (for instance) as we want.

We are now ready to establish our approximation theorem.

Theorem 2.5. (a) Let $f \in \mathcal{C}\left(\{0,1\}^{\mathbb{N}}\right)$ and $\epsilon>0$. There exist positive integers $K$, $S$ and $M$ so that if $k \geq K, s \geq S$ and $m$ is a positive integer multiple of $M$, then there are $g \in \mathcal{C}\left(\{0,1\}^{\mathbb{N}}\right)$ and a partition $\mathcal{P}$ of $\{0,1\}^{\mathbb{N}}$ with

$$
\tilde{d}(f, g)<\epsilon \quad \text { and } \operatorname{mesh}(\mathcal{P})<\epsilon,
$$

such that the digraph $\operatorname{Gr}(g, \mathcal{P})$ consists of exactly $k$ disjoint balloons of type $(s, m)$. (b) Let $f \in \mathcal{H}\left(\{0,1\}^{\mathbb{N}}\right)$ and $\epsilon>0$. There exist positive integers $K, N, S$ and $M$ so that if $k \geq K, s \geq S$ and $n, m$ are positive integer multiples of $N, M$, respectively, then there are $g \in \mathcal{H}\left(\{0,1\}^{\mathbb{N}}\right)$ and a partition $\mathcal{P}$ of $\{0,1\}^{\mathbb{N}}$ with

$$
\tilde{d}(f, g)<\epsilon \quad \text { and } \operatorname{mesh}(\mathcal{P})<\epsilon,
$$

such that the digraph $\operatorname{Gr}(g, \mathcal{P})$ consists of exactly $k$ disjoint dumbbells of type $(n, s, m)$.

Proof. Fix $f \in \mathcal{C}\left(\{0,1\}^{\mathbb{N}}\right)$ (resp. $f \in \mathcal{H}\left(\{0,1\}^{\mathbb{N}}\right)$ ) and $\epsilon>0$. Using the uniform continuity of $f$, we choose a partition $\mathcal{Q}$ of $\{0,1\}^{\mathbb{N}}$ such that

$$
\operatorname{mesh}(\mathcal{Q})<\frac{\epsilon}{2} \quad \text { and } \operatorname{mesh}(f(\mathcal{Q}))<\frac{\epsilon}{2} .
$$

As $\operatorname{Gr}(f, \mathcal{Q})$ is a digraph without right ends (resp. a digraph without ends), we can associate to $\operatorname{Gr}(f, \mathcal{Q})$ positive integers $K, S$ and $M$ (resp. $K, N, S$ and $M$ ) so that the property described in part (a) (resp. in part (b)) of Theorem 2.4 holds. Let $k \geq K, s \geq S$ and $m$ be a positive integer multiple of $M$ (resp. $n, m$ be positive integer multiples of $N, M$, respectively). Then, a digraph $G$ consisting of $k$ disjoint balloons of type $(s, m)$ (resp. of $k$ disjoint dumbells of type $(n, s, m)$ ) admits a graph map $\phi$ onto $\operatorname{Gr}(f, \mathcal{Q})$. By Theorem 2.2 , there exist a refinement $\mathcal{P}$ of $\mathcal{Q}$ and $g \in \mathcal{C}\left(\{0,1\}^{\mathbb{N}}\right)\left(\right.$ resp. $\left.g \in \mathcal{H}\left(\{0,1\}^{\mathbb{N}}\right)\right)$ so that $\operatorname{Gr}(g, \mathcal{P})$ is isomorphic to $G$ and

$$
\tilde{d}(f, g) \leq \operatorname{mesh}(\mathcal{Q})+\operatorname{mesh}(f(\mathcal{Q}))<\epsilon .
$$

This completes the proof.

\section{Conjugacy Relation}

In this section we present some characterizations of when two continuous maps of the Cantor space are topologically conjugate to each other. In particular, we can apply these characterizations to homeomorphisms of the Cantor space. 
We begin by recalling the definition of topological conjugacy. Suppose $f$ and $g$ are self-maps of spaces $X$ and $Y$, respectively. We say that $f$ and $g$ are topologically conjugate if there is a homeomorphism $h$ from $X$ onto $Y$ such that $f=h^{-1} g h$. We note that topological conjugacy is an equivalence relation. If $f, g \in \mathcal{C}\left(\{0,1\}^{\mathbb{N}}\right)$ and $h \in \mathcal{H}\left(\{0,1\}^{\mathbb{N}}\right)$, then we simply say that $f$ and $g$ are conjugates.

In order to state our next result, let us introduce some terminology.

If $\left(\mathcal{P}_{n}\right)$ is a sequence of partitions of $\{0,1\}^{\mathbb{N}}$, then we say that $\left(\mathcal{P}_{n}\right)$ is null whenever $\operatorname{mesh}\left(\mathcal{P}_{n}\right) \rightarrow 0$, and we say that $\left(\mathcal{P}_{n}\right)$ is decreasing whenever $\mathcal{P}_{n+1}$ is a refinement of $\mathcal{P}_{n}$ for every $n \in \mathbb{N}$. Note that every null sequence of partitions of $\{0,1\}^{\mathbb{N}}$ has a decreasing (and null) subsequence.

Suppose $f, g \in \mathcal{C}\left(\{0,1\}^{\mathbb{N}}\right),\left(\mathcal{P}_{n}\right)$ and $\left(\mathcal{Q}_{n}\right)$ are decreasing null sequences of partitions of $\{0,1\}^{\mathbb{N}}$ and $\left(\nu_{n}\right)$ is a sequence of isomorphisms

$$
\nu_{n}: \operatorname{Gr}\left(f, \mathcal{P}_{n}\right) \rightarrow \operatorname{Gr}\left(g, \mathcal{Q}_{n}\right) .
$$

For each $a \in \mathcal{P}_{n}$, we define

$$
\nu_{m}(a)=\bigcup\left\{\nu_{m}(b): b \in \mathcal{P}_{m} \text { and } b \subset a\right\} \quad(m \geq n)
$$

and

$$
\tilde{\nu}_{n}(a)=\bigcup_{m \geq n} \nu_{m}(a)
$$

We say that the sequence $\left(\nu_{n}\right)$ commutes with refinements if the diagrams

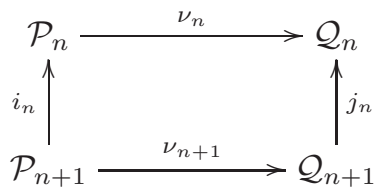

are commutative, where $i_{n}$ and $j_{n}$ denote the refinement maps. We have the following characterizations of this notion:

Proposition 3.1. With the above notations, the following assertions are equivalent:

(i) $\left(\nu_{n}\right)$ commutes with refinements;

(ii) $\nu_{m}(a)=\nu_{n}(a)$ whenever $m \geq n$ and $a \in \mathcal{P}_{n}$;

(iii) $\tilde{\nu}_{n}(a)=\nu_{n}(a)$ for every $n \in \mathbb{N}$ and $a \in \mathcal{P}_{n}$.

Proof. (i) $\Rightarrow$ (ii): Suppose $m \geq n$ and $a \in \mathcal{P}_{n}$. Since $\left(\nu_{n}\right)$ commutes with refinements, the diagram

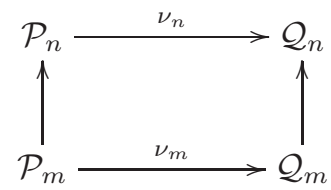

is commutative, where the up arrows indicate the refinement maps. Consequently,

$$
\nu_{m}(a) \subset \nu_{n}(a) .
$$

Conversely, take $\sigma \in \nu_{n}(a)$ and let $c \in \mathcal{Q}_{m}$ be such that $\sigma \in c$. Since $\nu_{m}$ is surjective, there exist $b \in \mathcal{P}_{m}$ such that $\nu_{m}(b)=c$. Let $a^{\prime} \in \mathcal{P}_{n}$ be such that $b \subset a^{\prime}$. By what we have just seen, $\nu_{m}\left(a^{\prime}\right) \subset \nu_{n}\left(a^{\prime}\right)$. Hence,

$$
\sigma \in c=\nu_{m}(b) \subset \nu_{m}\left(a^{\prime}\right) \subset \nu_{n}\left(a^{\prime}\right) .
$$


Since $\sigma \in \nu_{n}(a)$ and $\nu_{n}$ is injective, $a^{\prime}=a$. Thus, $\sigma \in \nu_{m}(a)$.

(ii) $\Rightarrow$ (iii): Obvious.

(iii) $\Rightarrow$ (i): Let $b \in \mathcal{P}_{n+1}$ and put $a=i_{n}(b) \in \mathcal{P}_{n}$. Then

$$
\nu_{n+1}(b) \subset \nu_{n+1}(a) \subset \tilde{\nu}_{n}(a)=\nu_{n}(a),
$$

by hypothesis. Hence, $j_{n}\left(\nu_{n+1}(b)\right)=\nu_{n}(a)=\nu_{n}\left(i_{n}(b)\right)$, as was to be shown.

We say that the sequence $\left(\nu_{n}\right)$ asymptotically commutes with refinements if

$$
\lim _{n \rightarrow \infty} \operatorname{mesh}\left(\left\{\tilde{\nu}_{n}(a): a \in \mathcal{P}_{n}\right\}\right)=0 .
$$

It follows from the previous proposition that if $\left(\nu_{n}\right)$ commutes with refinements, then both $\left(\nu_{n}\right)$ and $\left(\nu_{n}^{-1}\right)$ asymptotically commute with refinements.

Theorem 3.2. Let $f, g \in \mathcal{C}\left(\{0,1\}^{\mathbb{N}}\right)$. Then the following assertions are equivalent:

(i) $f$ and $g$ are conjugates;

(ii) There are decreasing null sequences $\left(\mathcal{P}_{n}\right)$ and $\left(\mathcal{Q}_{n}\right)$ of partitions of $\{0,1\}^{\mathbb{N}}$ and isomorphisms $\nu_{n}: \operatorname{Gr}\left(f, \mathcal{P}_{n}\right) \rightarrow \operatorname{Gr}\left(g, \mathcal{Q}_{n}\right)$ so that the sequence $\left(\nu_{n}\right)$ commutes with refinements;

(iii) There are decreasing null sequences $\left(\mathcal{P}_{n}\right)$ and $\left(\mathcal{Q}_{n}\right)$ of partitions of $\{0,1\}^{\mathbb{N}}$ and isomorphisms $\nu_{n}: \operatorname{Gr}\left(f, \mathcal{P}_{n}\right) \rightarrow \operatorname{Gr}\left(g, \mathcal{Q}_{n}\right)$ so that both $\left(\nu_{n}\right)$ and $\left(\nu_{n}^{-1}\right)$ asymptotically commute with refinements.

Proof. (i) $\Rightarrow$ (ii): Suppose $f=h^{-1} g h$ for a certain $h \in \mathcal{H}\left(\{0,1\}^{\mathbb{N}}\right)$. For each $n \in \mathbb{N}$, let $\mathcal{P}_{n}=\mathcal{B}_{n}$ and $\mathcal{Q}_{n}=\left\{h(a): a \in \mathcal{B}_{n}\right\}$. We now observe that for each $n \in \mathbb{N}$, $\operatorname{Gr}\left(f, \mathcal{P}_{n}\right)$ is isomorphic to $\operatorname{Gr}\left(g, \mathcal{Q}_{n}\right)$ by the map $\nu_{n}: a \in \mathcal{P}_{n} \mapsto h(a) \in \mathcal{Q}_{n}$. Indeed,

$$
\begin{aligned}
\overrightarrow{a b} \in \operatorname{Gr}\left(f, \mathcal{P}_{n}\right) & \Longleftrightarrow f(a) \cap b \neq \emptyset \\
& \Longleftrightarrow h f(a) \cap h(b) \neq \emptyset \\
& \Longleftrightarrow g h(a) \cap h(b) \neq \emptyset \\
& \Longleftrightarrow \overrightarrow{h(a) h(b)} \in \operatorname{Gr}\left(g, \mathcal{Q}_{n}\right) .
\end{aligned}
$$

Moreover, it is clear that the sequence $\left(\nu_{n}\right)$ commutes with refinements.

(ii) $\Rightarrow$ (iii): Obvious.

(iii) $\Rightarrow$ (i): For each $n \in \mathbb{N}$, we choose an $h_{n} \in \mathcal{H}\left(\{0,1\}^{\mathbb{N}}\right)$ such that

$$
h_{n}(a)=\nu_{n}(a) \text { for every } a \in \mathcal{P}_{n} \text {. }
$$

Then

$$
h_{n}^{-1}(c)=\nu_{n}^{-1}(c) \quad \text { for every } c \in \mathcal{Q}_{n} .
$$

We shall prove that $\left(h_{n}\right)$ is a Cauchy sequence in $\mathcal{C}\left(\{0,1\}^{\mathbb{N}}\right)$. For this purpose, let us fix $\epsilon>0$. Since $\left(\nu_{n}\right)$ asymptotically commutes with refinements, there exists $n_{0} \in \mathbb{N}$ such that

$$
\operatorname{mesh}\left(\left\{\tilde{\nu}_{n}(a): a \in \mathcal{P}_{n}\right\}\right)<\epsilon \quad \text { whenever } n \geq n_{0} .
$$

Take $\sigma \in\{0,1\}^{\mathbb{N}}$ and $m \geq n \geq n_{0}$. Let $a \in \mathcal{P}_{n}$ and $b \in \mathcal{P}_{m}$ be such that $\sigma \in a$ and $\sigma \in b$. Then,

$$
h_{n}(\sigma) \in \nu_{n}(a) \subset \tilde{\nu}_{n}(a) \quad \text { and } \quad h_{m}(\sigma) \in \nu_{m}(b) \subset \nu_{m}(a) \subset \tilde{\nu}_{n}(a),
$$

and so

$$
d\left(h_{m}(\sigma), h_{n}(\sigma)\right)<\epsilon \text {. }
$$


By completeness, the sequence $\left(h_{n}\right)$ must converge to a function $h$ in $\mathcal{C}\left(\{0,1\}^{\mathbb{N}}\right)$. Since $\left(\nu_{n}^{-1}\right)$ also asymptotically commutes with refinements, we may apply the same argument to the sequence $\left(h_{n}^{-1}\right)$ and conclude that this sequence converges to a function $t$ in $\mathcal{C}\left(\{0,1\}^{\mathbb{N}}\right)$. Since $h_{n} \circ h_{n}^{-1}=h_{n}^{-1} \circ h_{n}=I$ (the identity map of $\left.\{0,1\}^{\mathbb{N}}\right)$ for every $n \in \mathbb{N}, h \circ t=t \circ h=I$. Thus, $h \in \mathcal{H}\left(\{0,1\}^{\mathbb{N}}\right)$.

Now, given $\sigma \in\{0,1\}^{\mathbb{N}}$ and $n \in \mathbb{N}$, let $a, b \in \mathcal{P}_{n}$ be such that $\sigma \in a$ and $f(\sigma) \in b$. Then, $\overrightarrow{a b} \in \operatorname{Gr}\left(f, \mathcal{P}_{n}\right)$, which implies that $\overrightarrow{\nu_{n}(a) \nu_{n}(b)} \in \operatorname{Gr}\left(g, \mathcal{Q}_{n}\right)$, that is,

$$
g\left(\nu_{n}(a)\right) \cap \nu_{n}(b) \neq \emptyset .
$$

Since $h_{n} f(\sigma) \in \nu_{n}(b)$ and $g h_{n}(\sigma) \in g\left(\nu_{n}(a)\right)$, we obtain

$$
d\left(h_{n} f(\sigma), g h_{n}(\sigma)\right) \leq \operatorname{mesh}\left(\mathcal{Q}_{n}\right)+\operatorname{mesh}\left(g\left(\mathcal{Q}_{n}\right)\right)
$$

Hence, by letting $n \rightarrow \infty$, we conclude that $h f=g h$, that is, $f=h^{-1} g h$.

In order to state our next characterizations of the conjugacy relation, we need to introduce some further terminology.

Suppose $f, g \in \mathcal{C}\left(\{0,1\}^{\mathbb{N}}\right),\left(\mathcal{P}_{n}\right)$ and $\left(\mathcal{Q}_{n}\right)$ are decreasing null sequences of partitions of $\{0,1\}^{\mathbb{N}}$ and $\left(\nu_{n}\right)$ is a sequence of surjective graph maps with

$$
\nu_{n}: \operatorname{Gr}\left(f, \mathcal{P}_{n}\right) \rightarrow \operatorname{Gr}\left(g, \mathcal{Q}_{n}\right) \text { for odd } n
$$

and

$$
\nu_{n}: \operatorname{Gr}\left(g, \mathcal{Q}_{n}\right) \rightarrow \operatorname{Gr}\left(f, \mathcal{P}_{n}\right) \text { for even } n .
$$

For each odd $n$ and each $a \in \mathcal{P}_{n}$, we define

$$
\begin{aligned}
& \nu_{m}(a)=\bigcup\left\{\nu_{m}(b): b \in \mathcal{P}_{m} \text { and } b \subset a\right\} \quad(m \geq n, m \text { odd }), \\
& \nu_{m}^{-1}(a)=\bigcup\left\{c: c \in \mathcal{Q}_{m} \text { and } \nu_{m}(c) \subset a\right\} \quad(m>n, m \text { even })
\end{aligned}
$$

and

$$
\bar{\nu}_{n}(a)=\bigcup\left\{\nu_{m}(a): m \geq n, m \text { odd }\right\} \cup \bigcup\left\{\nu_{m}^{-1}(a): m>n, m \text { even }\right\} .
$$

Analogously, we define $\nu_{m}(c)(m \geq n, m$ even $), \nu_{m}^{-1}(c)(m>n, m$ odd $)$ and $\bar{\nu}_{n}(c)$ for even $n$ and $c \in \mathcal{Q}_{n}$. We say that the sequence $\left(\nu_{n}\right)$ commutes with refinements if the diagrams
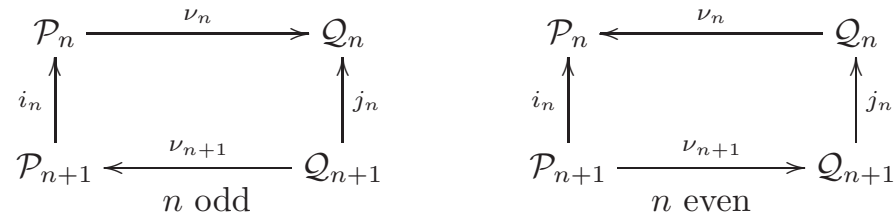

are commutative, where $i_{n}$ and $j_{n}$ denote the refinement maps. We have the following characterizations of this concept:

Proposition 3.3. With the above notations, the following assertions are equivalent:

(i) $\left(\nu_{n}\right)$ commutes with refinements;

(ii) The following inclusions hold:

- $\nu_{m}(a) \subset \nu_{n}(a)$ whenever $n$ is odd, $m$ is odd, $m \geq n$ and $a \in \mathcal{P}_{n}$.

- $\nu_{m}^{-1}(a) \subset \nu_{n}(a)$ whenever $n$ is odd, $m$ is even, $m>n$ and $a \in \mathcal{P}_{n}$.

- $\nu_{m}(c) \subset \nu_{n}(c)$ whenever $n$ is even, $m$ is even, $m \geq n$ and $c \in \mathcal{Q}_{n}$.

- $\nu_{m}^{-1}(c) \subset \nu_{n}(c)$ whenever $n$ is even, $m$ is odd, $m>n$ and $c \in \mathcal{Q}_{n}$.

(iii) The following equalities hold: 
- $\bar{\nu}_{n}(a)=\nu_{n}(a)$ whenever $n$ is odd and $a \in \mathcal{P}_{n}$.

- $\bar{\nu}_{n}(c)=\nu_{n}(c)$ whenever $n$ is even and $c \in \mathcal{Q}_{n}$.

Proof. (i) $\Rightarrow$ (ii): Let $n, m, k \in \mathbb{N}$ be such that $n$ and $m$ are odd, $k$ is even, $m \geq n$ and $k>n$. Since $\left(\nu_{n}\right)$ commutes with refinements, the diagrams
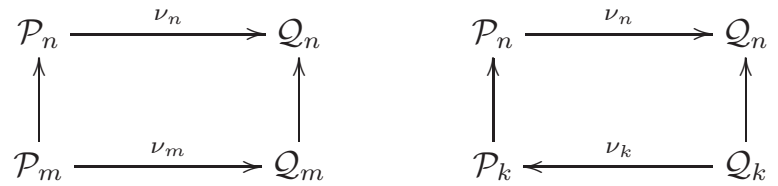

are commutative, where the up arrows indicate the refinement maps. This implies that $\nu_{m}(a) \subset \nu_{n}(a)$ and $\nu_{k}^{-1}(a) \subset \nu_{n}(a)$ for every $a \in \mathcal{P}_{n}$, which proves the first two inclusions in (ii). The other two inclusions are proved in a similar way.

(ii) $\Rightarrow$ (iii): Obvious.

(iii) $\Rightarrow$ (i): Assume $n$ odd and let us prove that

$$
j_{n}=\nu_{n} \circ i_{n} \circ \nu_{n+1} .
$$

Take $c \in \mathcal{Q}_{n+1}$ and put $b=\nu_{n+1}(c) \in \mathcal{P}_{n+1}$ and $a=i_{n}(b) \in \mathcal{P}_{n}$. Since $n+1$ is even and $\nu_{n+1}(c)=b \subset a$, we have that

$$
c \subset\left(\nu_{n+1}\right)^{-1}(a) \subset \bar{\nu}_{n}(a)=\nu_{n}(a) .
$$

Thus, $j_{n}(c)=\nu_{n}(a)$, that is, $j_{n}(c)=\nu_{n}\left(i_{n}\left(\nu_{n+1}(c)\right)\right)$. For $n$ even the proof is analogous.

We say that the sequence $\left(\nu_{n}\right)$ asymptotically commutes with refinements if

$$
\lim _{n \rightarrow \infty} \operatorname{mesh}\left(\left\{\bar{\nu}_{2 n-1}(a): a \in \mathcal{P}_{2 n-1}\right\}\right)=0
$$

and

$$
\lim _{n \rightarrow \infty} \operatorname{mesh}\left(\left\{\bar{\nu}_{2 n}(c): c \in \mathcal{Q}_{2 n}\right\}\right)=0 .
$$

By the previous proposition, if $\left(\nu_{n}\right)$ commutes with refinements, then $\left(\nu_{n}\right)$ asymptotically commutes with refinements.

Theorem 3.4. Let $f, g \in \mathcal{C}\left(\{0,1\}^{\mathbb{N}}\right)$. Then the following assertions are equivalent:

(i) $f$ and $g$ are conjugates;

(ii) There are decreasing null sequences $\left(\mathcal{P}_{n}\right)$ and $\left(\mathcal{Q}_{n}\right)$ of partitions of $\{0,1\}^{\mathbb{N}}$ and surjective graph maps $\nu_{2 n-1}: \operatorname{Gr}\left(f, \mathcal{P}_{2 n-1}\right) \rightarrow \operatorname{Gr}\left(g, \mathcal{Q}_{2 n-1}\right)$ and $\nu_{2 n}$ : $\operatorname{Gr}\left(g, \mathcal{Q}_{2 n}\right) \rightarrow \operatorname{Gr}\left(f, \mathcal{P}_{2 n}\right)$ so that the sequence $\left(\nu_{n}\right)$ commutes with refinements;

(iii) There are decreasing null sequences $\left(\mathcal{P}_{n}\right)$ and $\left(\mathcal{Q}_{n}\right)$ of partitions of $\{0,1\}^{\mathbb{N}}$ and surjective graph maps $\nu_{2 n-1}: \operatorname{Gr}\left(f, \mathcal{P}_{2 n-1}\right) \rightarrow \operatorname{Gr}\left(g, \mathcal{Q}_{2 n-1}\right)$ and $\nu_{2 n}:$ $\operatorname{Gr}\left(g, \mathcal{Q}_{2 n}\right) \rightarrow \operatorname{Gr}\left(f, \mathcal{P}_{2 n}\right)$ so that the sequence $\left(\nu_{n}\right)$ asymptotically commutes with refinements.

Proof. (i) $\Rightarrow$ (ii): By the implication (i) $\Rightarrow$ (ii) in Theorem 3.2 , there are decreasing null sequences $\left(\mathcal{P}_{n}\right)$ and $\left(\mathcal{Q}_{n}\right)$ of partitions of $\{0,1\}^{\mathbb{N}}$ and isomorphisms $\theta_{n}: \operatorname{Gr}\left(f, \mathcal{P}_{n}\right) \rightarrow \operatorname{Gr}\left(g, \mathcal{Q}_{n}\right)$ so that the sequence $\left(\theta_{n}\right)$ commutes with refinements. So, it is enough to define $\nu_{n}=\theta_{n}$ for odd $n$ and $\nu_{n}=\theta_{n}^{-1}$ for even $n$.

(ii) $\Rightarrow$ (iii): Obvious. 
(iii) $\Rightarrow$ (i): For each odd $n$ and each even $m$, we define

$$
\alpha_{n}=\operatorname{mesh}\left(\left\{\bar{\nu}_{n}(a): a \in \mathcal{P}_{n}\right\}\right) \quad \text { and } \quad \beta_{m}=\operatorname{mesh}\left(\left\{\bar{\nu}_{m}(c): c \in \mathcal{Q}_{m}\right\}\right) .
$$

Since $\left(\nu_{n}\right)$ asymptotically commutes with refinements,

$$
\left(\alpha_{n}\right)_{n \text { odd }} \rightarrow 0 \quad \text { and } \quad\left(\beta_{m}\right)_{m \text { even }} \rightarrow 0 .
$$

For each odd $n$, we choose an $h_{n} \in \mathcal{H}\left(\{0,1\}^{\mathbb{N}}\right)$ such that

$$
\bigcup_{a \in \nu_{n}^{-1}(c)} h_{n}(a)=c \quad \text { for every } c \in \mathcal{Q}_{n} .
$$

Note that

$$
h_{n}(a) \subset \nu_{n}(a) \text { for every } a \in \mathcal{P}_{n} .
$$

Given $\sigma \in\{0,1\}^{\mathbb{N}}$ and $m \geq n$ with $m$ and $n$ odd, let $a \in \mathcal{P}_{n}$ and $b \in \mathcal{P}_{m}$ be such that $\sigma \in a$ and $\sigma \in b$. Then

$$
h_{n}(\sigma) \in \nu_{n}(a) \subset \bar{\nu}_{n}(a) \quad \text { and } \quad h_{m}(\sigma) \in \nu_{m}(b) \subset \nu_{m}(a) \subset \bar{\nu}_{n}(a),
$$

and so $d\left(h_{m}(\sigma), h_{n}(\sigma)\right) \leq \alpha_{n}$. This proves that $\left(h_{n}\right)_{n}$ odd is a Cauchy sequence in $\mathcal{C}\left(\{0,1\}^{\mathbb{N}}\right)$ and so it converges to a certain $h \in \mathcal{C}\left(\{0,1\}^{\mathbb{N}}\right)$. Similarly, for each even $n$, we choose a $t_{n} \in \mathcal{H}\left(\{0,1\}^{\mathbb{N}}\right)$ such that

$$
\bigcup_{c \in \nu_{n}^{-1}(a)} t_{n}(c)=a \quad \text { for every } a \in \mathcal{P}_{n} .
$$

Then

$$
t_{n}(c) \subset \nu_{n}(c) \text { for every } c \in \mathcal{Q}_{n} .
$$

By arguing as before, we see that $\left(t_{n}\right)_{n}$ even converges to a certain $t \in \mathcal{C}\left(\{0,1\}^{\mathbb{N}}\right)$.

Now, let $\sigma \in\{0,1\}^{\mathbb{N}}$ and $n$ be odd. Let $c \in \mathcal{Q}_{n+1}$ and $a \in \mathcal{P}_{n}$ be such that $\sigma \in c$ and $t_{n+1}(\sigma) \in a$. As $t_{n+1}(\sigma) \in t_{n+1}(c) \subset \nu_{n+1}(c) \in \mathcal{P}_{n+1}$ and $t_{n+1}(\sigma) \in a \in \mathcal{P}_{n}$, we must have $\nu_{n+1}(c) \subset a$. Hence,

$$
\sigma \in c \subset\left(\nu_{n+1}\right)^{-1}(a) \subset \bar{\nu}_{n}(a) .
$$

On the other hand,

$$
h_{n}\left(t_{n+1}(\sigma)\right) \in h_{n}(a) \subset \nu_{n}(a) \subset \bar{\nu}_{n}(a) .
$$

Therefore, $d\left(h_{n}\left(t_{n+1}(\sigma)\right), \sigma\right) \leq \alpha_{n}$. This proves that $\left(h_{n} \circ t_{n+1}\right)_{n \text { odd }} \rightarrow I$, the identity map of $\{0,1\}^{\mathbb{N}}$. Analogously, $\left(t_{n} \circ h_{n+1}\right)_{n}$ even $\rightarrow I$. Thus, $h \circ t=t \circ h=I$, and so $h \in \mathcal{H}\left(\{0,1\}^{\mathbb{N}}\right)$.

Finally, by arguing exactly as in the last paragraph of the proof of Theorem 3.2 (but considering only odd $n$ ), we conclude that $f=h^{-1} \circ g \circ h$.

\section{Generics and Applichtions to Dynamics}

Suppose that $h \in \mathcal{H}\left(\{0,1\}^{\mathbb{N}}\right), \mathcal{P}$ is a partition of $\{0,1\}^{\mathbb{N}}$ and $D$ is a component of $\operatorname{Gr}(h, \mathcal{P})$ which is a dumbbell. Write

$$
D=\left\{u_{1}, \ldots, u_{r}\right\} \cup\left\{v_{1}, \ldots, v_{s}\right\} \cup\left\{w_{1}, \ldots, w_{t}\right\},
$$

with usual labeling. We say that $D$ contains a left loop of $h$ (resp. a right loop of $h$ ) if there is a nonempty clopen subset $a$ of $u_{1}$ (resp. of $w_{1}$ ) such that $h^{r}(a)=a$ (resp. $\left.h^{t}(a)=a\right)$.

By using our methods, we shall now give a simple geometric/graph theoretic description of the comeager conjugacy class of $\mathcal{H}\left(\{0,1\}^{\mathbb{N}}\right)$. 
Theorem 4.1. Let $S$ be the set of all $h \in \mathcal{H}\left(\{0,1\}^{\mathbb{N}}\right)$ with the following property: (P) For every $m \in \mathbb{N}$, there are a partition $\mathcal{P}$ of $\{0,1\}^{\mathbb{N}}$ of mesh $<1 / m$ and a multiple $q \in \mathbb{N}$ of $m$ such that every component of $\operatorname{Gr}(h, \mathcal{P})$ is a balanced dumbbell with plate weight $q$ ! that contains both a left and a right loop of $h$.

Then, $S$ is a comeager conjugacy class of $\mathcal{H}\left(\{0,1\}^{\mathbb{N}}\right)$.

Proof. For each $m \in \mathbb{N}$, let $S_{m}$ be the set of all $h \in \mathcal{H}\left(\{0,1\}^{\mathbb{N}}\right)$ that satisfies the property contained in (P) for this particular $m$. If we fix a partition $\mathcal{Q}$ of $\{0,1\}^{\mathbb{N}}$, then the map $f \rightarrow \operatorname{Gr}(f, \mathcal{Q})$ is locally constant on $\mathcal{C}\left(\{0,1\}^{\mathbb{N}}\right)$, because $f \sim_{\mathcal{Q}} g$ implies $\operatorname{Gr}(f, \mathcal{Q})=\operatorname{Gr}(g, \mathcal{Q})$. Moreover, if $a$ is clopen in $\{0,1\}^{\mathbb{N}}$ and $n \in \mathbb{N}$, then the condition $f^{n}(a) \subset a$ is an open condition on $\mathcal{C}\left(\{0,1\}^{\mathbb{N}}\right)$. By applying this to the inverse, we see that $f^{n}(a)=a$ is an open condition on $\mathcal{H}\left(\{0,1\}^{\mathbb{N}}\right)$. Therefore, each $S_{m}$ is open in $\mathcal{H}\left(\{0,1\}^{\mathbb{N}}\right)$. Let us prove that each $S_{m}$ is also dense in $\mathcal{H}\left(\{0,1\}^{\mathbb{N}}\right)$. For this purpose, fix $m \in \mathbb{N}, f \in \mathcal{H}\left(\{0,1\}^{\mathbb{N}}\right)$ and $\epsilon>0$. By applying the approximation theorem (Theorem 2.5(b)) with $\min \left\{\frac{\epsilon}{2}, \frac{1}{m}\right\}$ in place of $\epsilon$, we obtain positive integers $K, N, S$ and $M$ with the properties described in the theorem. Choose a multiple $q \geq 2$ of $m$ such that $q$ ! is a multiple of both $N$ and $M$. Then, with $k=K, s=S$ and $n=m=q$ !, Theorem 2.5 (b) gives us a $g \in \mathcal{H}\left(\{0,1\}^{\mathbb{N}}\right)$ and a partition $\mathcal{P}$ of $\{0,1\}^{\mathbb{N}}$ with

$$
\tilde{d}(f, g)<\frac{\epsilon}{2} \quad \text { and } \quad \operatorname{mesh}(\mathcal{P})<\min \left\{\frac{\epsilon}{2}, \frac{1}{m}\right\},
$$

such that $\operatorname{Gr}(g, \mathcal{P})$ is a digraph whose components are balanced dumbbells with plate weight $q$ !. Now, we define $h \in \mathcal{H}\left(\{0,1\}^{\mathbb{N}}\right)$ in the following way: for each component (dumbbell)

$$
D=\left\{u_{1}, \ldots, u_{q !}\right\} \cup\left\{v_{1}, \ldots, v_{s}\right\} \cup\left\{w_{1}, \ldots, w_{q}\right\}
$$

of $\operatorname{Gr}(g, \mathcal{P})$ (with usual labeling), we choose nonempty proper clopen subsets $a$ of $g^{-1}\left(u_{2}\right)$ and $b$ of $g\left(w_{q !}\right)$, define $h$ on $u_{q}$ ! so that

$$
h\left(g^{q !-1}(a)\right)=a \quad \text { and } \quad h\left(u_{q} \backslash g^{q !-1}(a)\right)=u_{1} \backslash a,
$$

define $h$ on $w_{q}$ ! so that

$$
h\left(g^{q !-1}(b)\right)=b \quad \text { and } \quad h\left(w_{q !} \backslash g^{q !-1}(b)\right)=g\left(w_{q !}\right) \backslash b,
$$

and put $h=g$ on the remaining vertices of $D$. Then, $\operatorname{Gr}(h, \mathcal{P})=\operatorname{Gr}(g, \mathcal{P})$ and each component (dumbbell) of $\operatorname{Gr}(h, \mathcal{P})$ contains both a left and a right loop of $h$, which shows that $h \in S_{m}$. Moreover, $\tilde{d}(g, h)<\frac{\epsilon}{2}$ because $\operatorname{mesh}(\mathcal{P})<\frac{\epsilon}{2}$. Therefore, $\tilde{d}(f, h)<\epsilon$, proving that $S_{m}$ is dense in $\mathcal{H}\left(\{0,1\}^{\mathbb{N}}\right)$. Thus, $S=\bigcap S_{m}$ is a comeager subset of $\mathcal{H}\left(\{0,1\}^{\mathbb{N}}\right)$.

In order to prove that $S$ is a conjugacy class, we shall begin by making several important remarks and introducing further terminology.

Suppose that $h \in \mathcal{H}\left(\{0,1\}^{\mathbb{N}}\right), \mathcal{P}$ is a partition of $\{0,1\}^{\mathbb{N}}$ and $D$ is a component of $\operatorname{Gr}(h, \mathcal{P})$ which is a dumbbell. Write

$$
D=\left\{u_{1}, \ldots, u_{r}\right\} \cup\left\{v_{1}, \ldots, v_{s}\right\} \cup\left\{w_{1}, \ldots, w_{t}\right\},
$$

with usual labeling. If we replace the set $u_{1}$ of $\mathcal{P}$ by the sets $h^{-1}\left(u_{2}\right)$ and $h^{-1}\left(v_{1}\right)$, we obtain a refinement $\mathcal{P}^{\prime}$ of $\mathcal{P}$ such that $\operatorname{Gr}\left(h, \mathcal{P}^{\prime}\right)$ has the following dumbbell as a component:

$$
D^{\prime}=\left\{u_{r}, h^{-1}\left(u_{2}\right), u_{2}, \ldots, u_{r-1}\right\} \cup\left\{h^{-1}\left(v_{1}\right), v_{1}, \ldots, v_{s}\right\} \cup\left\{w_{1}, \ldots, w_{t}\right\} .
$$


We call this procedure the method of increasing the bar of the dumbbell to the left. Note that this method doesn't change the plate weights but increase the bar length by 1 . Similarly, by breaking $w_{1}$ in the parts $h\left(v_{s}\right)$ and $h\left(w_{t}\right)$, we obtain a refinement $\mathcal{P}^{\prime \prime}$ of $\mathcal{P}$ such that $\operatorname{Gr}\left(h, \mathcal{P}^{\prime \prime}\right)$ has the following dumbbell as a component:

$$
D^{\prime \prime}=\left\{u_{1}, \ldots, u_{r}\right\} \cup\left\{v_{1}, \ldots, v_{s}, h\left(v_{s}\right)\right\} \cup\left\{w_{2}, \ldots, w_{t}, h\left(w_{t}\right)\right\} .
$$

This is called the method of increasing the bar of the dumbbell to the right. Hence, by applying these methods repeatedly, we can make the bar of the dumbbell $D$ increase to the left and/or to the right as much as we want. This remark will be quite important in the sequel.

Let $h \in \mathcal{H}\left(\{0,1\}^{\mathbb{N}}\right)$. We say that a partition $\mathcal{P}$ of $\{0,1\}^{\mathbb{N}}$ is $h$-regular if each component of $\operatorname{Gr}(h, \mathcal{P})$ is a balanced dumbbell that contains both a left and a right loop of $h$ and all components of $\operatorname{Gr}(h, \mathcal{P})$ have the same plate weight (denoted $w(h, \mathcal{P}))$. Note that the methods of increasing the bars of the dumbbells transform $h$-regular partitions in $h$-regular partitions. If $h \in S$ then there are $h$-regular partitions $\mathcal{P}$ such that $\operatorname{mesh}(\mathcal{P})$ is as small as we want and $w(h, \mathcal{P})$ is a multiple of any positive integer we want.

Suppose $\mathcal{P}$ and $\mathcal{P}^{\prime}$ are $h$-regular partitions. If mesh $\left(\mathcal{P}^{\prime}\right)$ is sufficiently small, then $\mathcal{P}^{\prime}$ is necessarily a refinement of $\mathcal{P}$. Assume that this is the case. Then, each component $D^{\prime}$ of $\operatorname{Gr}\left(h, \mathcal{P}^{\prime}\right)$ must be contained in some component $D$ of $\operatorname{Gr}(h, \mathcal{P})$, in the sense that the union of all vertices of $D^{\prime}$ is contained in the union of all vertices of $D$. Moreover, $w\left(h, \mathcal{P}^{\prime}\right)$ is necessarily a multiple of $w(h, \mathcal{P})$. Write

$$
D=\left\{u_{1}, \ldots, u_{q}\right\} \cup\left\{v_{1}, \ldots, v_{\ell}\right\} \cup\left\{w_{1}, \ldots, w_{q}\right\}
$$

and

$$
D^{\prime}=\left\{u_{1}^{\prime}, \ldots, u_{q^{\prime}}^{\prime}\right\} \cup\left\{v_{1}^{\prime}, \ldots, v_{\ell^{\prime}}^{\prime}\right\} \cup\left\{w_{1}^{\prime}, \ldots, w_{q^{\prime}}^{\prime}\right\}
$$

$\left(q=w(h, \mathcal{P})\right.$ and $\left.q^{\prime}=w\left(h, \mathcal{P}^{\prime}\right)\right)$, with usual labeling. There are three possibilities:

1) $\bigcup\left\{a: a \in D^{\prime}\right\} \subset u_{1} \cup \ldots \cup u_{q}$ :

By applying the methods of increasing the bar of $D^{\prime}$, we may assume $u_{1}^{\prime}, w_{1}^{\prime} \subset u_{1}$. Under this assumption, we say that $D^{\prime}$ is a subdumbbell of $D$ of type 1 .

2) $\left\{\left\{a: a \in D^{\prime}\right\} \subset w_{1} \cup \ldots \cup w_{q}\right.$ :

Similarly, we may assume $u_{1}^{\prime}, w_{1}^{\prime} \subset w_{1}$ in this case. Under this assumption, we say that $D^{\prime}$ is a subdumbbell of $D$ of type 2 .

3) $\bigcup\left\{a: a \in D^{\prime}\right\}$ meets $v_{1} \cup \ldots \cup v_{\ell}$ :

In this case, there must exist an integer $r \geq 0$ such that

$$
\begin{gathered}
v_{r+1}^{\prime} \subset v_{1}, v_{r+2}^{\prime} \subset v_{2}, \ldots, v_{r+\ell}^{\prime} \subset v_{\ell}, \\
u_{1}^{\prime} \cup \ldots \cup u_{q^{\prime}}^{\prime} \cup v_{1}^{\prime} \cup \ldots \cup v_{r}^{\prime} \subset u_{1} \cup \ldots \cup u_{q}
\end{gathered}
$$

and

$$
v_{r+\ell+1}^{\prime} \cup \ldots \cup v_{\ell^{\prime}}^{\prime} \cup w_{1}^{\prime} \cup \ldots \cup w_{q^{\prime}}^{\prime} \subset w_{1} \cup \ldots \cup w_{q} .
$$

By applying the methods of increasing the bar of $D^{\prime}$, we may assume $u_{1}^{\prime} \subset u_{1}$ and $w_{1}^{\prime} \subset w_{1}$. With this assumption, both the number $r$ of $v_{j}^{\prime}$ to the left of $v_{r+1}^{\prime}$ and the number $\ell^{\prime}-\ell-r$ of $v_{j}^{\prime}$ to the right of $v_{r+\ell}^{\prime}$ are multiples of $q$. Therefore, by applying the methods of increasing the bar of $D^{\prime}$ again, we may assume $r=\ell^{\prime}-\ell-r$. Geometrically, this equality give us symmetry: $v_{r+1}^{\prime}, \ldots, v_{r+\ell}^{\prime}$ lie in the center of the bar of $D^{\prime}$. Under these assumptions, we say that $D^{\prime}$ is a subdumbbell of $D$ of type 3 . 
The previous discussion suggests the following definition: if $\mathcal{P}$ and $\mathcal{P}^{\prime}$ are $h$ regular partitions, we say that $\mathcal{P}^{\prime}$ is an $h$-subpartition of $\mathcal{P}$ if $\mathcal{P}^{\prime}$ is a refinement of $\mathcal{P}$ and every component of $\operatorname{Gr}\left(h, \mathcal{P}^{\prime}\right)$ is a subdumbbell (of type 1, 2 or 3 ) of a component of $\operatorname{Gr}(h, \mathcal{P})$. We have seen that if $h \in S$, then every $h$-regular partition $\mathcal{P}$ has $h$-subpartitions $\mathcal{P}^{\prime}$ such that $\operatorname{mesh}\left(\mathcal{P}^{\prime}\right)$ is as small as we want and $w\left(h, \mathcal{P}^{\prime}\right)$ is a multiple of any positive integer we want.

Suppose $\mathcal{P}^{\prime}$ is an $h$-subpartition of $\mathcal{P}$ and let $D$ be a component of $\operatorname{Gr}(h, \mathcal{P})$. Then $D$ can be thought of as the union of its subdumbbells relative to $\mathcal{P}^{\prime}$. Clearly, there must exist at least one subdumbbell of $D$ of type 3 . Since $D$ has both a left and a right loop of $h$, there must also exist subdumbbells of $D$ of types 1 and 2 provided $\operatorname{mesh}\left(\mathcal{P}^{\prime}\right)$ is sufficiently small. More precisely, we can make the number of subdumbbells of $D$ of type 1 (resp. type 2, type 3 ) as large as we want by choosing $\mathcal{P}^{\prime}$ with $\operatorname{mesh}\left(\mathcal{P}^{\prime}\right)$ small enough.

We are now in position to prove that $S$ is a conjugacy class. If $f \in S$ and $g \in \mathcal{H}\left(\{0,1\}^{\mathbb{N}}\right)$ is conjugate to $f$, then it is easy to verify that $g \in S$. Let us fix $f, g \in S$. It remains to prove that $f$ and $g$ are conjugates. In view of Theorem 3.4 it is enough to construct sequences $\left(\mathcal{P}_{n}\right),\left(\mathcal{Q}_{n}\right)$ and $\left(\nu_{n}\right)$ with the properties described in part (ii) of the theorem.

In order to construct $\mathcal{P}_{1}, \mathcal{Q}_{1}$ and $\nu_{1}$, we begin by taking a $g$-regular partition $\mathcal{Q}_{1}$ with $\operatorname{mesh}\left(\mathcal{Q}_{1}\right)<1$. Then, we take an $f$-regular partition $\mathcal{P}_{1}$ such that $\operatorname{mesh}\left(\mathcal{P}_{1}\right)<$ $1, w\left(f, \mathcal{P}_{1}\right)$ is a multiple of $w\left(g, \mathcal{Q}_{1}\right)$ and the set $A$ of all components of $\operatorname{Gr}\left(f, \mathcal{P}_{1}\right)$ has cardinality greather than or equal to that of the set $B$ of all components of $\operatorname{Gr}\left(g, \mathcal{Q}_{1}\right)$. By applying the methods of increasing the bars of the dumbbells, we may assume that all dumbbells in $A \cup B$ have the same bar length. Choose a surjection $\phi: A \rightarrow B$. For each $D \in A$, we define $\nu_{1}$ on $D$ as the unique surjection from $D$ onto $\phi(D)$ that maps the bar of $D$ onto the bar of $\phi(D)$ and satisfies the relation

$$
\overrightarrow{a b} \in \operatorname{Gr}\left(f, \mathcal{P}_{1}\right) \Longrightarrow \overrightarrow{\nu_{1}(a) \nu_{1}(b)} \in \operatorname{Gr}\left(g, \mathcal{Q}_{1}\right) \quad(a, b \in D) .
$$

In this way, we obtain a surjective graph map $\nu_{1}: \operatorname{Gr}\left(f, \mathcal{P}_{1}\right) \rightarrow \operatorname{Gr}\left(g, \mathcal{Q}_{1}\right)$.

In order to construct $\mathcal{P}_{2}, \mathcal{Q}_{2}$ and $\nu_{2}$, we begin by taking an $f$-subpartition $\mathcal{P}_{2}$ of $\mathcal{P}_{1}$ such that $\operatorname{mesh}\left(\mathcal{P}_{2}\right)<1 / 2$ and every component of $\operatorname{Gr}\left(f, \mathcal{P}_{1}\right)$ has subdumbbells of types 1, 2 and 3 relative to $\mathcal{P}_{2}$. Then, we take a $g$-subpartition $\mathcal{Q}_{2}$ of $\mathcal{Q}_{1}$ such that $\operatorname{mesh}\left(\mathcal{Q}_{2}\right)<1 / 2, w\left(g, \mathcal{Q}_{2}\right)$ is a multiple of $w\left(f, \mathcal{P}_{2}\right)$ and every component of $\operatorname{Gr}\left(g, \mathcal{Q}_{1}\right)$ has subdumbbells of types 1,2 and 3 relative to $\mathcal{Q}_{2}$. Fix a component $D$ of $\operatorname{Gr}\left(g, \mathcal{Q}_{1}\right)$ and let $\left\{D_{1}, \ldots, D_{r}\right\}$ be the set of all components $D_{j}$ of $\operatorname{Gr}\left(f, \mathcal{P}_{1}\right)$ such that $\nu_{1}\left(D_{j}\right)=D$. We can divide the set of all subdumbbells $D^{\prime}$ of $D$ relative to $\mathcal{Q}_{2}$ in three sets $A_{1}, A_{2}$ and $A_{3}$, according to whether $D^{\prime}$ is of type 1,2 or 3 , respectively. Similarly, for each $1 \leq j \leq r$, the set of all subdumbbells of $D_{j}$ relative to $\mathcal{P}_{2}$ is a union of three disjoint sets $B_{j, 1}, B_{j, 2}$ and $B_{j, 3}$. We may assume that $\mathcal{Q}_{2}$ was chosen so that

$$
\operatorname{Card} A_{i} \geq \operatorname{Card}\left(B_{1, i} \cup \ldots \cup B_{r, i}\right) \quad \text { for } i=1,2,3 .
$$

Hence, we may choose a surjection $\phi_{i}: A_{i} \rightarrow B_{1, i} \cup \ldots \cup B_{r, i}(i=1,2,3)$. Moreover, by using the facts that the dumbbells $D, D_{1}, \ldots, D_{r}$ have the same bar length and that each number in the finite sequence $w\left(g, \mathcal{Q}_{2}\right), w\left(f, \mathcal{P}_{2}\right), w\left(f, \mathcal{P}_{1}\right), w\left(g, \mathcal{Q}_{1}\right)$ is a multiple of its successor, and by applying the methods of increasing the bars of the dumbbells, we may assume that all dumbbells in $A_{i} \cup B_{1, i} \cup \ldots \cup B_{r, i}$ have the same bar length (but the bar length may depend on $i$ ). Now, for each $i \in\{1,2,3\}$ 
and each $D^{\prime} \in A_{i}$, we define $\nu_{2}$ on $D^{\prime}$ as the unique surjection from $D^{\prime}$ onto $\phi_{i}\left(D^{\prime}\right)$ that maps the bar of $D^{\prime}$ onto the bar of $\phi_{i}\left(D^{\prime}\right)$ and satisfies the relation

$$
\overrightarrow{a b} \in \operatorname{Gr}\left(g, \mathcal{Q}_{2}\right) \Longrightarrow \overrightarrow{\nu_{2}(a) \nu_{2}(b)} \in \operatorname{Gr}\left(f, \mathcal{P}_{2}\right) \quad\left(a, b \in D^{\prime}\right) .
$$

With this definition, note that

$$
j_{1}(a)=\nu_{1}\left(i_{1}\left(\nu_{2}(a)\right)\right) \text { for every } a \in D^{\prime},
$$

where $i_{1}: \mathcal{P}_{2} \rightarrow \mathcal{P}_{1}$ and $j_{1}: \mathcal{Q}_{2} \rightarrow \mathcal{Q}_{1}$ are the refinement maps. Indeed, it is easy to see that this is true by using the following elementary arithmetic fact: if $n_{1}, n_{2}, m_{1}, m_{2} \in \mathbb{N}, m_{1}$ divides $n_{1}, n_{1}$ divides $n_{2}$ and $n_{2}$ divides $m_{2}$, then the diagram

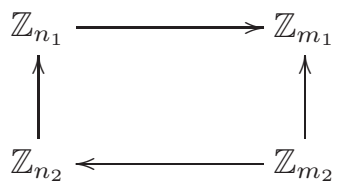

is commutative, where $\mathbb{Z}_{n} \rightarrow \mathbb{Z}_{m}$ denotes the $\bmod m$ map. By defining $\nu_{2}$ in this way for each component $D$ of $\operatorname{Gr}\left(g, \mathcal{Q}_{1}\right)$, we obtain a surjective graph map $\nu_{2}: \operatorname{Gr}\left(g, \mathcal{Q}_{2}\right) \rightarrow \operatorname{Gr}\left(f, \mathcal{P}_{2}\right)$ such that $j_{1}=\nu_{1} \circ i_{1} \circ \nu_{2}$.

Now, we apply exactly the same procedure to construct $\mathcal{P}_{3}, \mathcal{Q}_{3}$ and $\nu_{3}$ (but with $\mathcal{P}_{3}$ and $\mathcal{Q}_{3}$ in place of $\mathcal{Q}_{2}$ and $\mathcal{P}_{2}$, respectively), and so on. This completes the proof that $f$ and $g$ are conjugates.

Our goal in the remaining of the present section is to show how the description of the comeager conjugacy class of $\mathcal{H}\left(\{0,1\}^{\mathbb{N}}\right)$ given by Theorem 4.1 can be used to establish with little effort very precise properties of the homeomorphisms of this class. This will make clear that this description is quite useful and very easy to use. We observe that any comeager dynamical property of an element of $\mathcal{H}\left(\{0,1\}^{\mathbb{N}}\right)$ is automatically satisfied by all elements of the comeager conjugacy class. Let us also mention that several of these properties can also be derived from the explicit construction of Akin, Glasner and Weiss [3].

Let us begin by considering the notion of chaos. As mentioned in the introduction, there are several different notions of chaos, Li-Yorke chaos being the weakest of them. Let us recall that a pair $(x, y)$ is a Li-Yorke pair for a function $f$ if

$$
\liminf _{n \rightarrow \infty} d\left(f^{n}(x), f^{n}(y)\right)=0 \quad \text { and } \quad \limsup _{n \rightarrow \infty} d\left(f^{n}(x), f^{n}(y)\right)>0 .
$$

A function $f$ is Li-Yorke chaotic if there is an uncountable set $S$ such that $(x, y)$ is a Li-Yorke pair for $f$ whenever $x$ and $y$ are distinct points in $S$. It was proved in [15] that the set of homeomorphisms in $\mathcal{H}\left(\{0,1\}^{\mathbb{N}}\right)$ which have topological entropy zero is comeager in $\mathcal{H}\left(\{0,1\}^{\mathbb{N}}\right)$. Hence, a homeomorphism chosen at "random" is not chaotic in the sense of entropy. We shall now see that the elements of the comeager conjugacy class are not even Li-Yorke chaotic. In fact, a much stronger assertion can be made.

Theorem 4.2. No element of the comeager conjugacy class of $\mathcal{H}\left(\{0,1\}^{\mathbb{N}}\right)$ has a Li-Yorke pair.

Proof. Let $h$ be an element of the comeager conjugacy class of $\mathcal{H}\left(\{0,1\}^{\mathbb{N}}\right)$. Then, $h$ satisfies property $(\mathrm{P})$ of Theorem 4.1. Assume $(\sigma, \tau)$ is a Li-Yorke pair for $h$ and 
choose $m \in \mathbb{N}$ such that

$$
\frac{1}{m}<\limsup _{n \rightarrow \infty} d\left(h^{n}(\sigma), h^{n}(\tau)\right) .
$$

Then, there is a partition $\mathcal{P}$ of $\{0,1\}^{\mathbb{N}}$ of mesh $<1 / m$ such that every component of $\operatorname{Gr}(h, \mathcal{P})$ is a dumbbell. By our choice of $m$, there must exist infinitely many $n$ 's such that $h^{n}(\sigma)$ and $h^{n}(\tau)$ lie in different sets of $\mathcal{P}$. On the other hand, since $\liminf _{n \rightarrow \infty} d\left(h^{n}(\sigma), h^{n}(\tau)\right)=0$, there must exist infinitely many $n$ 's such that both $h^{n}(\sigma)$ and $h^{n}(\tau)$ lie in the same set of $\mathcal{P}$. But this impossible since every component of $\operatorname{Gr}(h, \mathcal{P})$ is a dumbbell and two points in the same vertex of such a dumbbell can be mapped into different vertices only once.

Let $h: X \rightarrow X$ be a homeomorphism, where $X$ is a metric space. A sequence $\left(x_{n}\right)_{n \in \mathbb{Z}}$ is a $\delta$-pseudotrajectory $(\delta>0)$ of $h$ if

$$
d\left(h\left(x_{n}\right), x_{n+1}\right) \leq \delta \text { for every } n \in \mathbb{Z} .
$$

Recall that $h$ is said to have the shadowing property [9, 10 (also called pseudoorbit tracing property) if for every $\epsilon>0$ there exists $\delta>0$ such that every $\delta$ pseudotrajectory $\left(x_{n}\right)_{n \in \mathbb{Z}}$ of $h$ is $\epsilon$-shadowed by a real trajectory of $h$, i.e., there exists $x \in X$ such that

$$
d\left(x_{n}, h^{n}(x)\right)<\epsilon \text { for every } n \in \mathbb{Z} .
$$

Recall also that $h$ is said to have the weak shadowing property [12] if for every $\epsilon>0$ there exists $\delta>0$ such that for every $\delta$-pseudotrajectory $\left(x_{n}\right)_{n \in \mathbb{Z}}$ of $h$ there exists $x \in X$ such that the set $\left\{x_{n}: n \in \mathbb{Z}\right\}$ is contained in the $\epsilon$-neighborhood of the orbit $\left\{h^{n}(x): n \in \mathbb{Z}\right\}$. It was proved in [19] that there is a comeager subset of $\mathcal{H}\left(\{0,1\}^{\mathbb{N}}\right)$, each element of which has the weak shadowing property. We shall now see that it actually has the shadowing property.

Theorem 4.3. Each element of the comeager conjugacy class of $\mathcal{H}\left(\{0,1\}^{\mathbb{N}}\right)$ has the shadowing property.

Proof. Let $h$ be an element of the comeager conjugacy class of $\mathcal{H}\left(\{0,1\}^{\mathbb{N}}\right)$. Fix $\epsilon>0$ and choose $m \in \mathbb{N}$ with $1 / m<\epsilon$. Then, there is a partition $\mathcal{P}$ of $\{0,1\}^{\mathbb{N}}$ of mesh $<1 / m$ such that every component of $\operatorname{Gr}(h, \mathcal{P})$ is a dumbbell. Let $\delta$ be the minimum distance between two distinct elements of $\mathcal{P}$. To each sequence $X=\left(\sigma_{n}\right)_{n \in \mathbb{Z}}$ in $\{0,1\}^{\mathbb{N}}$, we associate the sequence $S(X)=\left(S_{n}(X)\right)_{n \in \mathbb{Z}}$ in $\mathcal{P}$ which satisfies $\sigma_{n} \in S_{n}(X)$ for every $n \in \mathbb{Z}$. Let $W=\left(\tau_{n}\right)_{n \in \mathbb{Z}}$ be a $\delta$-pseudotrajectory of $h$ and let us prove that it can be $\epsilon$-shadowed by a real trajectory of $h$. Let

$$
D=\left\{u_{1}, \ldots, u_{r}\right\} \cup\left\{v_{1}, \ldots, v_{s}\right\} \cup\left\{w_{1}, \ldots, w_{t}\right\}
$$

be the dumbbell in $\operatorname{Gr}(h, \mathcal{P})$ (with usual labeling) that contains a vertex containing $\tau_{0}$. By our choice of $\delta$, there are only three possibilities for the sequence $S(W)$, namely:

(1) $\left(\ldots, u_{1}, \ldots, u_{r}, u_{1}, \ldots, u_{r}, v_{1}, \ldots, v_{s}, w_{1}, \ldots, w_{t}, w_{1}, \ldots, w_{t}, \ldots\right)$, or

(2) $\left(\ldots, u_{1}, \ldots, u_{r}, u_{1}, \ldots, u_{r}, u_{1}, \ldots, u_{r}, \ldots\right)$, or

(3) $\left(\ldots, w_{1}, \ldots, w_{t}, w_{1}, \ldots, w_{t}, w_{1}, \ldots, w_{t}, \ldots\right)$.

Since $\mathcal{P}$ has mesh $<\epsilon$, it is enough to find real trajectories

$$
X=\left(h^{n}(x)\right)_{n \in \mathbb{Z}}, \quad Y=\left(h^{n}(y)\right)_{n \in \mathbb{Z}} \quad \text { and } \quad Z=\left(h^{n}(z)\right)_{n \in \mathbb{Z}}
$$


such that $S(X), S(Y)$ and $S(Z)$ are of type (1), (2) and (3), respectively. For the first case, it is enough to get any $x$ in a vertex of the bar of $D$. For the second case, note that

$$
u_{1} \supset h^{-r}\left(u_{1}\right) \supset h^{-2 r}\left(u_{1}\right) \supset \cdots,
$$

so that the intersection $\bigcap_{n=0}^{\infty} h^{-n r}\left(u_{1}\right)$ is nonempty; then take any $y$ in this intersection. Finally, since

$$
w_{1} \backslash h\left(v_{s}\right) \supset h^{t}\left(w_{1} \backslash h\left(v_{s}\right)\right) \supset h^{2 t}\left(w_{1} \backslash h\left(v_{s}\right)\right) \supset \cdots,
$$

we may take any $z \in \bigcap_{n=0}^{\infty} h^{n t}\left(w_{1} \backslash h\left(v_{s}\right)\right)$.

Given $\alpha \in(\mathbb{N} \backslash\{1\})^{\mathbb{N}}$, consider the product space

$$
\Delta_{\alpha}=\prod_{i=1}^{\infty} \mathbb{Z}_{\alpha(i)},
$$

where $\mathbb{Z}_{k}=\{0, \ldots, k-1\}$ with the discrete topology. Note that $\Delta_{\alpha}$ is homeomorphic to the Cantor space. We define an operation of addition on $\Delta_{\alpha}$ in the following way: if $\left(x_{1}, x_{2}, \ldots\right)$ and $\left(y_{1}, y_{2}, \ldots\right)$ are in $\Delta_{\alpha}$, then

$$
\left(x_{1}, x_{2}, \ldots\right)+\left(y_{1}, y_{2}, \ldots\right)=\left(z_{1}, z_{2}, \ldots\right),
$$

where $z_{1}=x_{1}+y_{1} \bmod \alpha(1)$ and, in general, $z_{i}$ is defined recursively as $z_{i}=$ $x_{i}+y_{i}+\epsilon_{i-1} \bmod \alpha(i)$ where $\epsilon_{i-1}=0$ if $x_{i-1}+y_{i-1}+\epsilon_{i-2}<\alpha(i-1)$ and $\epsilon_{i-1}=1$ otherwise. If we let $f_{\alpha}$ be the " $+1 "$ map, that is,

$$
f_{\alpha}\left(x_{1}, x_{2}, \ldots\right)=\left(x_{1}, x_{2}, \ldots\right)+(1,0,0, \ldots),
$$

then $\left(\Delta_{\alpha}, f_{\alpha}\right)$ is a dynamical system known as a solenoid, adding machine or odometer. We also define a function $M_{\alpha}$ from the set of primes into $\{0,1,2, \ldots, \infty\}$ by

$$
M_{\alpha}(p)=\sum_{i=1}^{\infty} n(i),
$$

where $n(i)$ is the largest integer such that $p^{n(i)}$ divides $\alpha(i)$. The following beautiful characterization of odometers up to topological conjugacy is due to Buescu and Stewart [1]:

Let $\alpha, \beta \in(\mathbb{N} \backslash\{1\})^{\mathbb{N}}$. Then $f_{\alpha}$ and $f_{\beta}$ are topologically conjugate if and only if $M_{\alpha}=M_{\beta}$.

When $M_{\alpha}(p)=\infty$ for every $p, f_{\alpha}$ is said to be an universal odometer. It follows from the above-mentioned result that any two universal odometers are topologically conjugate.

We shall need the following result from [8]:

Let $\alpha \in(\mathbb{N} \backslash\{1\})^{\mathbb{N}}$ and $m_{i}=\alpha(1) \alpha(2) \cdots \alpha(i)$ for each $i$. Let $f: X \rightarrow X$ be $a$ continuous map of a compact topological space $X$. Then $f$ is topologically conjugate to $f_{\alpha}$ if and only if (1)-(3) hold:

(1) For each positive integer $i$, there is a cover $\mathcal{P}_{i}$ of $X$ consisting of $m_{i}$ nonempty pairwise disjoint clopen sets which are cyclically permuted by $f$.

(2) For each positive integer $i, \mathcal{P}_{i+1}$ refines $\mathcal{P}_{i}$.

(3) If $W_{1} \supset W_{2} \supset W_{3} \supset \cdots$ is a nested sequence with $W_{i} \in \mathcal{P}_{i}$ for each $i$, then $\bigcap_{i=1}^{\infty} W_{i}$ consists of a single point. 
Let us also recall that the $\omega$-limit set $\omega(x, f)$ of $f$ at $x$ is the set of all limit points of the sequence $\left(f^{n}(x)\right)_{n \in \mathbb{N}}$.

Theorem 4.4. Let $h$ be an element of the comeager conjugacy class of $\mathcal{H}\left(\{0,1\}^{\mathbb{N}}\right)$. Then, the restriction of $h$ to every $\omega$-limit set $\omega(\sigma, h)$ is topologically conjugate to the universal odometer.

Proof. Since $h$ satisfies property $(\mathrm{P})$, we can construct inductively a sequence $\left(\mathcal{P}_{m}\right)_{m \in \mathbb{N}}$ of partitions of $\{0,1\}^{\mathbb{N}}$ and a sequence $\left(q_{m}\right)_{m \in \mathbb{N}}$ of natural numbers so that the following properties hold for every $m \in \mathbb{N}$ :

- $\operatorname{mesh}\left(\mathcal{P}_{m}\right)<1 / m$

- $\mathcal{P}_{m+1}$ refines $\mathcal{P}_{m}$;

- $q_{m+1}$ is a multiple of $m q_{m}$;

- every component of $\operatorname{Gr}\left(h, \mathcal{P}_{m}\right)$ is a balanced dumbbell of plate weight $q_{m}$ !

Let $\sigma \in\{0,1\}^{\mathbb{N}}$ and consider the $\omega$-limit set $\omega(\sigma, h)$. For each $m \in \mathbb{N}, \sigma$ belongs to a vertex of a certain dumbbell $D_{m}$ in $\operatorname{Gr}\left(h, \mathcal{P}_{m}\right)$. Then, $\omega(\sigma, h)$ must be contained in one of the loops of $D_{m}$, for each $m \in \mathbb{N}$. Thus, it follows from the above-mentioned result from [8] that $\left.h\right|_{\omega(\sigma, h)}: \omega(\sigma, h) \rightarrow \omega(\sigma, h)$ is topologically conjugate to $f_{\alpha}$, where

$$
\alpha=\left(q_{1} !, \frac{q_{2} !}{q_{1} !}, \frac{q_{3} !}{q_{2} !}, \frac{q_{4} !}{q_{3} !}, \ldots\right) .
$$

Since $m$ ! divides $\frac{q_{m+1} !}{q_{m} !}$ for every $m \in \mathbb{N}, f_{\alpha}$ is an universal odometer.

Given a continuous map $f: X \rightarrow X$, where $X$ is a metric space, we shall denote by $P(f)$ (resp. $R(f), \Omega(f), C R(f)$ ) the set of all periodic points (resp. recurrent points, nonwandering points, chain recurrent points) of $f[5]$.

Theorem 4.5. Let $h$ be an element of the comeager conjugacy class of $\mathcal{H}\left(\{0,1\}^{\mathbb{N}}\right)$. Then, we have that:

(a) $P(h)$ is empty.

(b) $R(h)=\Omega(h)=C R(h)$.

(c) $R(h)$ is a Cantor set with empty interior in $\{0,1\}^{\mathbb{N}}$.

Proof. (a): Obvious.

(b): Since $R(h) \subset \Omega(h) \subset C R(h)$, let us prove that $C R(h) \subset R(h)$. For this purpose, suppose $\sigma \notin R(h)$. Then, there is an $m \in \mathbb{N}$ such that the set

$$
\left\{n \in \mathbb{N}: d\left(h^{n}(\sigma), \sigma\right)<1 / m\right\}
$$

is finite. Let $\mathcal{P}$ be a partition of $\{0,1\}^{\mathbb{N}}$ of mesh $<1 / m$ such that every component of $\operatorname{Gr}(h, \mathcal{P})$ is a dumbbell. Let $D$ be the dumbbell in $\operatorname{Gr}(h, \mathcal{P})$ which contains a vertex containing $\sigma$ and write

$$
D=\left\{u_{1}, \ldots, u_{r}\right\} \cup\left\{v_{1}, \ldots, v_{s}\right\} \cup\left\{w_{1}, \ldots, w_{t}\right\}
$$

with usual labeling. By our choice of $m, \sigma$ must belong either to the bar $\left\{v_{1}, \ldots, v_{s}\right\}$ or to the loop $\left\{u_{1}, \ldots, u_{r}\right\}$ and, in this last case, its trajectory must leave this loop at some moment. Both possibilities imply that $\sigma \notin C R(h)$.

(c): Let us now prove that $R(h)$ is a Cantor set. Since $R(h)=\Omega(h)$, which is closed and nonempty, it is enough to show that $R(h)$ has no isolated point. So, take a point $\tau \in R(h)$. Then $\tau \in \omega(\tau, h)$. Since $\omega(\tau, h) \subset \Omega(h)=R(h)$ and $\omega(\tau, h)$ is a Cantor set (Theorem 4.4), $\tau$ is not an isolated point of $R(h)$. Finally, suppose $A$ is 
a nonempty open set of $\{0,1\}^{\mathbb{N}}$ which is contained in $R(h)$ and fix $\sigma \in A$. Let $\mathcal{P}$ and $D$ be as in the proof of (b), where $m$ is chosen so big that the vertex $v$ of $D$ containing $\sigma$ must be contained in $A$. It is easy to see that every point in every set of the collection

$$
\left\{h^{-r}\left(v_{1}\right), \ldots, h^{-2}\left(v_{1}\right), h^{-1}\left(v_{1}\right), v_{1}, \ldots, v_{s}, h\left(v_{s}\right), h^{2}\left(v_{s}\right), \ldots, h^{t}\left(v_{s}\right)\right\}
$$

is nonrecurrent. Thus, every vertex of $D$ contains a nonrecurrent point, contradicting the fact that $v$ is contained in $A$.

To the best of our knowledge, the fact that the set of all $h \in \mathcal{H}\left(\{0,1\}^{\mathbb{N}}\right)$ which have no periodic point is comeager in $\mathcal{H}\left(\{0,1\}^{\mathbb{N}}\right)$ first appeared in [4].

Recall that a mapping $f$ from a metric space $X$ into itself is said to be equicontinuous at a point $x \in X$ if for every $\epsilon>0$ there exists $\delta>0$ such that

$$
d(y, x)<\delta \quad \Longrightarrow \quad d\left(f^{n}(y), f^{n}(x)\right)<\epsilon \text { for every } n \geq 0 .
$$

Moreover, $f$ is said to be chain continuous at $x$ [1, 6] if for every $\epsilon>0$ there exists $\delta>0$ such that for any choice of points

$$
x_{0} \in B(x ; \delta), x_{1} \in B\left(f\left(x_{0}\right) ; \delta\right), x_{2} \in B\left(f\left(x_{1}\right) ; \delta\right), \ldots,
$$

we have that

$$
d\left(x_{n}, f^{n}(x)\right)<\epsilon \text { for every } n \geq 0 .
$$

Of course, chain continuity is a much stronger property than equicontinuity.

Theorem 4.6. Let $h$ be an element of the comeager conjugacy class of $\mathcal{H}\left(\{0,1\}^{\mathbb{N}}\right)$. Then, $h$ is chain continuous at every nonrecurrent point and so it is chain continuous at every point of a dense open set, but it is not equicontinuous at each point of an uncountable set.

Proof. Let $\sigma$ be a nonrecurrent point of $h$ and fix $\epsilon>0$. Choose $m \in \mathbb{N}$ such that $1 / m<\epsilon$ and the set

$$
\left\{n \in \mathbb{N}: d\left(h^{n}(\sigma), \sigma\right)<1 / m\right\}
$$

is finite. Let $\mathcal{P}$ be a partition of $\{0,1\}^{\mathbb{N}}$ of mesh $<1 / m$ such that every component of $\operatorname{Gr}(h, \mathcal{P})$ is a dumbbell and let

$$
D=\left\{u_{1}, \ldots, u_{r}\right\} \cup\left\{v_{1}, \ldots, v_{s}\right\} \cup\left\{w_{1}, \ldots, w_{t}\right\}
$$

be the dumbbell in $\operatorname{Gr}(h, \mathcal{P})$ (with usual labeling) that contains a vertex containing $\sigma$. We want to find a $\delta>0$ such that the relations $\sigma_{0} \in B(\sigma ; \delta), \sigma_{1} \in B\left(h\left(\sigma_{0}\right) ; \delta\right)$, $\sigma_{2} \in B\left(h\left(\sigma_{1}\right) ; \delta\right), \ldots$ imply $d\left(\sigma_{n}, h^{n}(\sigma)\right)<\epsilon$ for every $n \geq 0$. If $\sigma \in v_{1} \cup \ldots \cup v_{s} \cup$ $w_{1} \cup \ldots \cup w_{t}$, then it is enough to choose $0<\delta<\epsilon$ smaller than the minimum distance between two distinct elements of $\mathcal{P}$. If $\sigma$ is in the loop $\left\{u_{1}, \ldots, u_{r}\right\}$, our choice of $m$ implies that the trajectory of $\sigma$ must eventually leave this loop, and so it is clear that we can also find such a $\delta$ in this case. Thus, we have proved that $h$ is chain continuous at every point of the set $\{0,1\}^{\mathbb{N}} \backslash R(h)$, which is open and dense in view of Theorem 4.5 .

Let us now prove the last assertion. Since $u_{1} \supset h^{-r}\left(u_{1}\right) \supset h^{-2 r}\left(u_{1}\right) \supset \cdots$, the closed set

$$
Y=\bigcap_{n=0}^{\infty} h^{-n r}\left(u_{1}\right)
$$


is nonempty and satisfies $h^{r}(Y)=Y$. If $Y$ were open, it would follow from the inclusions $u_{1} \backslash Y \supset h^{-r}\left(u_{1} \backslash Y\right) \supset h^{-2 r}\left(u_{1} \backslash Y\right) \supset \cdots$ that the intersection $\bigcap_{n=0}^{\infty} h^{-n r}\left(u_{1} \backslash Y\right)$ is nonempty, which is impossible. Thus, the closed set

$$
Z=\overline{u_{1} \backslash Y} \cap Y
$$

is nonempty. Moreover, $h^{r}(Z)=Z$. If $\sigma \in Z$ then the trajectory of $\sigma$ remains in the loop $\left\{u_{1}, \ldots, u_{r}\right\}$ forever (because $\sigma \in Y$ ) but as close to $\sigma$ as we want there are points of $u_{1} \backslash Y$ (because $\sigma \in \overline{u_{1} \backslash Y}$ ) and the trajectories of these points eventually go to the bar of the dumbbell $D$. This proves that $h$ is not equicontinuous at $\sigma$. Thus, $h$ is not equicontinuous at each point of the set

$$
Z \cup h(Z) \cup \ldots \cup h^{r-1}(Z)
$$

Since this set is closed and invariant under $h$, it contains the $\omega$-limit set of each of its elements, and so it is uncountable in view of Theorem 4.4

\section{The Case of Continuous Maps}

Suppose that $f \in \mathcal{C}\left(\{0,1\}^{\mathbb{N}}\right), \mathcal{P}$ is a partition of $\{0,1\}^{\mathbb{N}}$ and $B$ is a component of $\operatorname{Gr}(f, \mathcal{P})$ which is a balloon. Write

$$
B=\left\{v_{1}, \ldots, v_{s}\right\} \cup\left\{w_{1}, \ldots, w_{t}\right\},
$$

with usual labeling. We say that $B$ is strict relative to $f$ if $f\left(v_{i}\right) \subsetneq v_{i+1}$ for every $1 \leq i<s, f\left(w_{j}\right) \subsetneq w_{j+1}$ for every $1 \leq j<t$, and $f\left(v_{s}\right) \cup f\left(w_{t}\right) \subsetneq w_{1}$.

Surprisingly enough, we shall now prove that there is a comeager subset of $\mathcal{C}\left(\{0,1\}^{\mathbb{N}}\right)$ such that any two elements of this set are conjugate to each other.

Theorem 5.1. Let $S$ be the set of all $f \in \mathcal{C}\left(\{0,1\}^{\mathbb{N}}\right)$ with the following property:

(Q) For every $m \in \mathbb{N}$, there are a partition $\mathcal{P}$ of $\{0,1\}^{\mathbb{N}}$ of mesh $<1 / m$ and a multiple $q \in \mathbb{N}$ of $m$ such that every component of $\operatorname{Gr}(f, \mathcal{P})$ is a balloon of type $(q !, q !)$ which is strict relative to $f$.

Then, $S$ is a comeager subset of $\mathcal{C}\left(\{0,1\}^{\mathbb{N}}\right)$ such that any two of its elements are conjugate to each other.

Proof. For each $m \in \mathbb{N}$, let $S_{m}$ be the set of all $f \in \mathcal{C}\left(\{0,1\}^{\mathbb{N}}\right)$ that satisfies the property contained in (Q) for this particular $m$. Clearly, each $S_{m}$ is open in $\mathcal{C}\left(\{0,1\}^{\mathbb{N}}\right)$. In order to prove that each $S_{m}$ is also dense $\mathcal{C}\left(\{0,1\}^{\mathbb{N}}\right)$, let us fix $m \in \mathbb{N}, f \in \mathcal{C}\left(\{0,1\}^{\mathbb{N}}\right)$ and $\epsilon>0$. It follows from Theorem 2.5)(a) that there are $g \in \mathcal{C}\left(\{0,1\}^{\mathbb{N}}\right)$ with $\tilde{d}(f, g)<\frac{\epsilon}{2}$, a partition $\mathcal{P}$ of $\{0,1\}^{\mathbb{N}}$ of mesh $<\min \left\{\frac{\epsilon}{2}, \frac{1}{m}\right\}$, and a multiple $q \in \mathbb{N}$ of $m$ such that $\operatorname{Gr}(g, \mathcal{P})$ is a digraph whose components are balloons of type $(q !, q !)$. If $\psi:\{0,1\}^{\mathbb{N}} \rightarrow\{0,1\}^{\mathbb{N}}$ maps each $a \in \mathcal{P}$ to a single point of $a$, then $\psi$ is continuous. Moreover, $\psi \circ g \sim_{\mathcal{P}} g$, which implies that $\operatorname{Gr}(\psi \circ g, \mathcal{P})=\operatorname{Gr}(g, \mathcal{P})$ and $\tilde{d}(\psi \circ g, g) \leq \operatorname{mesh}(\mathcal{P})<\frac{\epsilon}{2}$ (and so $\left.\tilde{d}(\psi \circ g, f)<\epsilon\right)$. Since $\psi \circ g$ has finite range, each component (balloon) of $\operatorname{Gr}(\psi \circ g, \mathcal{P})$ is strict relative to $\psi \circ g$. Hence, $\psi \circ g \in S_{m}$, proving that $S_{m}$ is dense in $\mathcal{C}\left(\{0,1\}^{\mathbb{N}}\right)$. Thus, $S=\bigcap S_{m}$ is a comeager subset of $\mathcal{C}\left(\{0,1\}^{\mathbb{N}}\right)$.

Let $f \in \mathcal{C}\left(\{0,1\}^{\mathbb{N}}\right)$. We say that a partition $\mathcal{P}$ of $\{0,1\}^{\mathbb{N}}$ is $f$-admissible if there is a $k \in \mathbb{N}$ such that every component of $\operatorname{Gr}(f, \mathcal{P})$ is a balloon of type $(k, k)$ which is strict relative to $f$. In this case, we denote this number $k$ by $b(f, \mathcal{P})$. If $f \in S$ then there are $f$-admissible partitions $\mathcal{P}$ such that $\operatorname{mesh}(\mathcal{P})$ is as small as we want and $b(f, \mathcal{P})$ is a multiple of any positive integer we want. 
Suppose $\mathcal{P}$ and $\mathcal{P}^{\prime}$ are $f$-admissible partitions. If $\operatorname{mesh}\left(\mathcal{P}^{\prime}\right)$ is sufficiently small, then $\mathcal{P}^{\prime}$ is necessarily a refinement of $\mathcal{P}$. Assume that this is the case. Then, each component $B^{\prime}$ of $\operatorname{Gr}\left(f, \mathcal{P}^{\prime}\right)$ must be contained in some component $B$ of $\operatorname{Gr}(f, \mathcal{P})$, in the sense that the union of all vertices of $B^{\prime}$ is contained in the union of all vertices of $B$. Moreover, $b\left(f, \mathcal{P}^{\prime}\right)$ is necessarily a multiple of $b(f, \mathcal{P})$. Let $B$ be a component of $\operatorname{Gr}(f, \mathcal{P})$. We say that a component $B^{\prime}$ of $\operatorname{Gr}\left(f, \mathcal{P}^{\prime}\right)$ is a subballoon of $B$ of type $u$ if the initial vertex of $B^{\prime}$ is contained in the vertex $u$ of $B$. With this definition, $B$ can be thought of as the union of its subballoons relative to $\mathcal{P}^{\prime}$. Since the balloon $B$ is strict relative to $f$, there must exist subballoons of $B$ of every type $u \in B$ provided $\operatorname{mesh}\left(\mathcal{P}^{\prime}\right)$ is sufficiently small. More precisely, we can make the number of subballoons of $B$ of each type $u$ as large as we want by choosing $\mathcal{P}^{\prime}$ with $\operatorname{mesh}\left(\mathcal{P}^{\prime}\right)$ small enough.

If $f \in S$ and $g \in \mathcal{C}\left(\{0,1\}^{\mathbb{N}}\right)$ is conjugate to $f$, then it is easy to verify that $g \in S$. Take $f, g \in S$ and let us prove that $f$ and $g$ are conjugates. It is enough to construct sequences $\left(\mathcal{P}_{n}\right),\left(\mathcal{Q}_{n}\right)$ and $\left(\nu_{n}\right)$ with the properties described in part (ii) of Theorem 3.4

We begin by taking a $g$-admissible partition $\mathcal{Q}_{1}$ with $\operatorname{mesh}\left(\mathcal{Q}_{1}\right)<1$. Then, we take an $f$-admissible partition $\mathcal{P}_{1}$ such that $\operatorname{mesh}\left(\mathcal{P}_{1}\right)<1, b\left(f, \mathcal{P}_{1}\right)$ is a multiple of $b\left(g, \mathcal{Q}_{1}\right)$ and the set $X$ of all components of $\operatorname{Gr}\left(f, \mathcal{P}_{1}\right)$ has cardinality greather than or equal to that of the set $Y$ of all components of $\operatorname{Gr}\left(g, \mathcal{Q}_{1}\right)$. Finally, we choose a surjection $\phi: X \rightarrow Y$ and, for each $B \in X$, we define $\nu_{1}$ on $B$ as the unique surjection from $B$ onto $\phi(B)$ that maps the initial vertex of $B$ to the initial vertex of $\phi(B)$ and satisfies the relation

$$
\overrightarrow{a b} \in \operatorname{Gr}\left(f, \mathcal{P}_{1}\right) \Longrightarrow \overrightarrow{\nu_{1}(a) \nu_{1}(b)} \in \operatorname{Gr}\left(g, \mathcal{Q}_{1}\right) \quad(a, b \in B) .
$$

In this way, we obtain a surjective graph map $\nu_{1}: \operatorname{Gr}\left(f, \mathcal{P}_{1}\right) \rightarrow \operatorname{Gr}\left(g, \mathcal{Q}_{1}\right)$.

Now, we take an $f$-admissible partition $\mathcal{P}_{2}$ such that $\mathcal{P}_{2}$ refines $\mathcal{P}_{1}, \operatorname{mesh}\left(\mathcal{P}_{2}\right)<$ $1 / 2$ and every component of $\operatorname{Gr}\left(f, \mathcal{P}_{1}\right)$ has subballoons of every type relative to $\mathcal{P}_{2}$. Then, we take a $g$-admissible partition $\mathcal{Q}_{2}$ such that $\mathcal{Q}_{2}$ refines $\mathcal{Q}_{1}, \operatorname{mesh}\left(\mathcal{Q}_{2}\right)<1 / 2$, $b\left(g, \mathcal{Q}_{2}\right)$ is a multiple of $b\left(f, \mathcal{P}_{2}\right)$ and every component of $\operatorname{Gr}\left(g, \mathcal{Q}_{1}\right)$ has subballoons of every type relative to $\mathcal{Q}_{2}$. Let us fix a component $B$ of $\operatorname{Gr}\left(g, \mathcal{Q}_{1}\right)$ and let $\left\{B_{1}, \ldots, B_{r}\right\}$ be the set of all components $B_{k}$ of $\operatorname{Gr}\left(f, \mathcal{P}_{1}\right)$ such that $\nu_{1}\left(B_{k}\right)=B$. For each $u \in B$, let $X_{u}$ be the set of all subballoons of $B$ (relative to $\mathcal{Q}_{2}$ ) of type $u$. Moreover, for each $1 \leq k \leq r$, let $Y_{k, u}$ be the set of all subballoons of $B_{k}$ (relative to $\mathcal{P}_{2}$ ) of type $v$ for some $v \in \nu_{1}^{-1}(\{u\})$. We may assume that $\mathcal{Q}_{2}$ was chosen so that

$$
\operatorname{Card} X_{u} \geq \operatorname{Card}\left(Y_{1, u} \cup \ldots \cup Y_{r, u}\right) \quad \text { for every } u \in B \text {. }
$$

Hence, we may choose a surjection $\phi_{u}: X_{u} \rightarrow Y_{1, u} \cup \ldots \cup Y_{r, u}(u \in B)$. Finally, for each $u \in B$ and each $B^{\prime} \in X_{u}$, we define $\nu_{2}$ on $B^{\prime}$ as the unique surjection from $B^{\prime}$ onto $\phi_{u}\left(B^{\prime}\right)$ that maps the initial vertex of $B^{\prime}$ to the initial vertex of $\phi_{u}\left(B^{\prime}\right)$ and satisfies the relation

$$
\overrightarrow{a b} \in \operatorname{Gr}\left(g, \mathcal{Q}_{2}\right) \Longrightarrow \overrightarrow{\nu_{2}(a) \nu_{2}(b)} \in \operatorname{Gr}\left(f, \mathcal{P}_{2}\right) \quad\left(a, b \in B^{\prime}\right) .
$$

We claim that

$$
(*) \quad j_{1}(a)=\nu_{1}\left(i_{1}\left(\nu_{2}(a)\right)\right) \quad \text { for every } a \in B^{\prime},
$$

where $i_{1}: \mathcal{P}_{2} \rightarrow \mathcal{P}_{1}$ and $j_{1}: \mathcal{Q}_{2} \rightarrow \mathcal{Q}_{1}$ are the refinement maps. In fact, let us first consider the initial vertex $c$ of $B^{\prime}$. Let $k \in\{1, \ldots, r\}$ be such that $\phi_{u}\left(B^{\prime}\right) \in Y_{k, u}$. 
Since $j_{1}(c)=u$ (because $\left.B^{\prime} \in X_{u}\right)$ and $i_{1}\left(\nu_{2}(c)\right) \in \nu_{1}^{-1}(\{u\})$ (because $\nu_{2}(c)$ is the initial vertex of $\phi_{u}\left(B^{\prime}\right)$ and $\left.\phi_{u}\left(B^{\prime}\right) \in Y_{k, u}\right)$, it follows that $c$ satisfies the equality in $(*)$. Now, let us assume that a certain vertex $a$ of $B^{\prime}$ satisfies the equality in (*). Let $b$ be the unique vertex of $B^{\prime}$ such that $\overrightarrow{a b} \in B^{\prime}$. Since

$$
\begin{aligned}
& \overrightarrow{a b} \in B^{\prime} \Longrightarrow \overrightarrow{j_{1}(a) j_{1}(b)} \in B, \\
& \overrightarrow{a b} \in B^{\prime} \Longrightarrow \overrightarrow{\nu_{2}(a) \nu_{2}(b)} \in \phi_{u}\left(B^{\prime}\right) \\
& \Longrightarrow \overrightarrow{i_{1}\left(\nu_{2}(a)\right) i_{1}\left(\nu_{2}(b)\right)} \in B_{k} \\
& \Longrightarrow \overrightarrow{\nu_{1}\left(i_{1}\left(\nu_{2}(a)\right)\right) \nu_{1}\left(i_{1}\left(\nu_{2}(b)\right)\right)} \in B \text {, }
\end{aligned}
$$

and we are assuming that $j_{1}(a)=\nu_{1}\left(i_{1}\left(\nu_{2}(a)\right)\right)$, it follows that $b$ also satisfies the equality in $(*)$. By induction, we see that $(*)$ holds. Thus, by defining $\nu_{2}$ in this way for each component $B$ of $\operatorname{Gr}\left(g, \mathcal{Q}_{1}\right)$, we obtain a surjective graph map $\nu_{2}: \operatorname{Gr}\left(g, \mathcal{Q}_{2}\right) \rightarrow \operatorname{Gr}\left(f, \mathcal{P}_{2}\right)$ such that $j_{1}=\nu_{1} \circ i_{1} \circ \nu_{2}$.

Now, we apply exactly the same procedure to construct $\mathcal{P}_{3}, \mathcal{Q}_{3}$ and $\nu_{3}$ (but with $\mathcal{P}_{3}$ and $\mathcal{Q}_{3}$ in place of $\mathcal{Q}_{2}$ and $\mathcal{P}_{2}$, respectively), and so on. This completes the proof.

Let us now establish some applications to dynamics.

It was proved in 13 that the set of elements of $\mathcal{C}\left(\{0,1\}^{\mathbb{N}}\right)$ which have zero topological entropy and no periodic points is comeager in $\mathcal{C}\left(\{0,1\}^{\mathbb{N}}\right)$. Hence, an element chosen at "random" from $\mathcal{C}\left(\{0,1\}^{\mathbb{N}}\right)$ is not chaotic in the sense of entropy nor in the sense of Devaney. We show something much stronger below.

Theorem 5.2. There is a comeager subset of $\mathcal{C}\left(\{0,1\}^{\mathbb{N}}\right)$, no element of which has a Li-Yorke pair.

Proof. Let $f \in \mathcal{C}\left(\{0,1\}^{\mathbb{N}}\right)$ satisfy property (Q) of Theorem [5.1] Suppose that $\sigma, \tau \in\{0,1\}^{\mathbb{N}}$ satisfy

$$
\liminf _{n \rightarrow \infty} d\left(f^{n}(\sigma), f^{n}(\tau)\right)=0
$$

Fix $\epsilon>0$ and choose $m \in \mathbb{N}$ such that $1 / m<\epsilon$. Then, there is a partition $\mathcal{P}$ of $\{0,1\}^{\mathbb{N}}$ of mesh $<1 / m$ such that every component of $\operatorname{Gr}(f, \mathcal{P})$ is a balloon. Moreover, there must exists an $n_{0} \in \mathbb{N}$ such that both $f^{n_{0}}(\sigma)$ and $f^{n_{0}}(\tau)$ lie in the same vertex $a$ of $\operatorname{Gr}(f, \mathcal{P})$. Let $B$ be the component of $\operatorname{Gr}(f, \mathcal{P})$ that contains $a$. Since $B$ is a balloon, $f$ maps each vertex of $B$ into a vertex of $B$. Hence, both $f^{n}(\sigma)$ and $f^{n}(\tau)$ lie in the same vertex of $B$, so that $d\left(f^{n}(\sigma), f^{n}(\tau)\right)<\epsilon$, for each $n \geq n_{0}$. This proves that

$$
\lim _{n \rightarrow \infty} d\left(f^{n}(\sigma), f^{n}(\tau)\right)=0
$$

and so $(\sigma, \tau)$ is not a Li-Yorke pair for $f$.

In contrast to the case of homeomorphisms (Theorem 4.6), we have the following

Theorem 5.3. The set of maps $f$ of $\mathcal{C}\left(\{0,1\}^{\mathbb{N}}\right)$ such that $f$ is chain continuous at every point is comeager in $\mathcal{C}\left(\{0,1\}^{\mathbb{N}}\right)$.

Proof. Let $f \in \mathcal{C}\left(\{0,1\}^{\mathbb{N}}\right)$ satisfy property (Q). Fix $\epsilon>0$ and choose $m \in \mathbb{N}$ such that $1 / m<\epsilon$. Then, there is a partition $\mathcal{P}$ of $\{0,1\}^{\mathbb{N}}$ of mesh $<1 / m$ such that every component of $\operatorname{Gr}(f, \mathcal{P})$ is a balloon. Let $\delta$ be the minimum distance between 
two distinct elements of $\mathcal{P}$. Given $\sigma \in\{0,1\}^{\mathbb{N}}$, let $B$ be the component of $\operatorname{Gr}(f, \mathcal{P})$ which contains a vertex containing $\sigma$. Since $B$ is a balloon, $f$ maps each vertex of $B$ into a vertex of $B$. Hence, if $\sigma_{0} \in B(\sigma ; \delta), \sigma_{1} \in B\left(f\left(\sigma_{0}\right) ; \delta\right), \sigma_{2} \in B\left(f\left(\sigma_{1}\right) ; \delta\right), \ldots$, then both $\sigma_{n}$ and $f^{n}(\sigma)$ lie in the same vertex of $B$, so that $d\left(\sigma_{n}, f^{n}(\sigma)\right)<\epsilon$, for every $n \geq 0$.

It was proved in 14 that there is a comeager subset of $\mathcal{C}\left(\{0,1\}^{\mathbb{N}}\right)$ such that each element $f$ in this set has property that the restriction of $f$ to the $\omega$-limit set $\omega(\sigma, f)$ is topologically conjugate to the universal odometer for a comeager set of $\sigma \in\{0,1\}^{\mathbb{N}}$. The next result tell us that this actually holds for every point $\sigma \in\{0,1\}^{\mathbb{N}}$.

Theorem 5.4. There is a comeager subset of $\mathcal{C}\left(\{0,1\}^{\mathbb{N}}\right)$ such that each $f$ in this set has the following property: The restriction of $f$ to every $\omega$-limit set $\omega(\sigma, f)$ is topologically conjugate to the universal odometer.

Proof. The proof of this result is similar to that of Theorem 4.4 and so we omit it.

Theorem 5.5. The set of all $f \in \mathcal{C}\left(\{0,1\}^{\mathbb{N}}\right)$ which satisfies the following properties is comeager in $\mathcal{C}\left(\{0,1\}^{\mathbb{N}}\right)$ :

(a) $P(f)$ is empty.

(b) $R(f)=\Omega(f)=C R(f)$.

(c) $R(f)$ is a Cantor set with empty interior in $f\left(\{0,1\}^{\mathbb{N}}\right)$.

Proof. Let $f \in \mathcal{C}\left(\{0,1\}^{\mathbb{N}}\right)$ satisfy property (Q).

(a): Obvious.

(b): The fact that $f$ is chain continuous at every point (Theorem 5.3) clearly implies that $C R(f) \subset R(f)$, and so (b) holds.

(c): If $\tau \in R(f)$ then $\tau \in \omega(\tau, f) \subset R(f)$, which implies that $\tau$ is not an isolated point of $R(f)$ since $\omega(\tau, f)$ is a Cantor set (Theorem [5.4). In view of (b), we conclude that $R(f)$ is a Cantor set. Finally, suppose that $U$ is a nonempty open set of $f\left(\{0,1\}^{\mathbb{N}}\right)$ which is contained in $R(f)$. Fix $\sigma \in U$ and let $V$ be an open set of $\{0,1\}^{\mathbb{N}}$ such that $U=V \cap f\left(\{0,1\}^{\mathbb{N}}\right)$. Let $\mathcal{P}$ be a partition of $\{0,1\}^{\mathbb{N}}$ such that every component of $\operatorname{Gr}(f, \mathcal{P})$ is a balloon and $\operatorname{mesh}(\mathcal{P})$ is so small that the vertex $v$ of $\operatorname{Gr}(f, \mathcal{P})$ containing $\sigma$ must be contained in $V$. Let

$$
B=\left\{v_{1}, \ldots, v_{s}\right\} \cup\left\{w_{1}, \ldots, w_{t}\right\}
$$

be the component (balloon) of $\operatorname{Gr}(f, \mathcal{P})$ which contains the vertex $v$. Since $\sigma \in$ $R(f), v$ must be one of the vertices $w_{1}, \ldots, w_{t}$; say $v=w_{j}$. Then

$$
f^{j}\left(v_{s}\right) \subset w_{j}=v \subset V \text { and } f^{j}\left(v_{s}\right) \cap R(f)=\emptyset,
$$

which implies that $f^{j}\left(v_{s}\right) \subset U \backslash R(f)=\emptyset$, a contradiction.

We remark that it was established in $\left[13\right.$ that the set of $f \in \mathcal{C}\left(\{0,1\}^{\mathbb{N}}\right)$ without periodic point forms a comeager subset of $\mathcal{C}\left(\{0,1\}^{\mathbb{N}}\right)$.

\section{ACKNOWLEDGEMENT}

The first author was partially supported by CAPES: Bolsista - Proc. $\mathrm{n}^{\circ}$ BEX 4012/11-9. 
The second author thanks the Mathematics Institute of Federal University of Rio de Janeiro for its support and hospitality during his one-month visit in 2011.

The authors are grateful to the anonymous referee for making several valuable changes which significantly improved the exposition of the paper.

\section{REFERENCES}

[1] E. Akin, On chain continuity, Discrete Contin. Dynam. Systems 2 (1996), no. 1, 111-120.

[2] E. Akin, E. Glasner, W. Huang, S. Shao and X. Ye, Sufficient conditions under which a transitive system is chaotic, Ergodic Theory Dynam. Systems 30 (2010), no. 5, 1277-1310.

[3] E. Akin, E. Glasner and B. Weiss, Generically there is but one self homeomorphism of the Cantor set, Trans. Amer. Math. Soc. 360 (2008), no. 7, 3613-3630.

[4] E. Akin, M. Hurley and J. Kennedy, Dynamics of topologically generic homeomorphisms, Mem. Amer. Math. Soc. 164 (2003), no. 783.

[5] N. Aoki and K. Hiraide, Topological Theory of Dynamical Systems - Recent Advances, NorthHolland, 1994.

[6] N. C. Bernardes Jr., On the predictability of discrete dynamical systems, Proc. Amer. Math. Soc. 130 (2002), no. 7, 1983-1992.

[7] F. Blanchard, E. Glasner, S. Kolyada and A. Maass, On Li-Yorke pairs, J. Reine Angew. Math. 547 (2002), 51-68.

[8] L. Block and J. Keesling, A characterization of adding machine maps, Topology Appl. 140 (2004), no. 2-3, 151-161.

[9] R. Bowen, Equilibrium states and the ergodic theory of Anosov diffeomorphisms, Lecture Notes in Mathematics 470, Springer-Verlag, 1975.

[10] R. Bowen, w-limit sets for Axiom A diffeomorphisms, J. Differential Equations 18 (1975), no. 2, 333-339.

[11] J. Buescu and I. Stewart, Lyapunov stability and adding machines, Ergodic Theory Dynam. Systems 15 (1995), 271-290.

[12] R. M. Corless and S. Yu Pilyugin, Approximate and real trajectories for generic dynamical systems, J. Math. Anal. Appl. 189 (1995), no. 2, 409-423.

[13] E. D'Aniello and U. B. Darji, Chaos among self-maps of the Cantor space, J. Math. Anal. Appl. 381 (2011), no. 2, 781-788.

[14] E. D'Aniello, U. B. Darji and T. H. Steele, Ubiquity of odometers in topological dynamical systems, Topology Appl. 156 (2008), no. 2, 240-245.

[15] E. Glasner and B. Weiss, The topological Rohlin property and topological entropy, Amer. J. Math. 123 (2001), no. 6, 1055-1070.

[16] M. Hochman, Genericity in topological dynamics, Ergodic Theory Dynam. Systems 28 (2008), no. 1, 125-165.

[17] A. Kwiatkowska, The group of homeomorphisms of the Cantor set has ample generics, preprint.

[18] A. S. Kechris and C. Rosendal, Turbulence, amalgamation, and generic automorphisms of homogeneous structures, Proc. London Math. Soc. (3) 94 (2007), no. 2, 302-350.

[19] M. Mazur, Weak shadowing for discrete dynamical systems on nonsmooth manifolds, J. Math. Anal. Appl. 281 (2003), no. 2, 657-662.

[20] J. K. Truss, Generic automorphisms of homogeneous structures, Proc. London Math. Soc. (3) 65 (1992), no. 1, 121-141.

(Bernardes) Instituto de Matemática, Universidade Federal do Rio de Janeiro, Caixa Postal 68530, Rio de Janeiro, RJ 21945-970, Brasil

E-mail address, Bernardes: bernardes@im.ufrj.br USA

(Darji) Department of Mathematics, University of Louisville, Louisville, KY 40292,

E-mail address, Darji: ubdarj01@louisville.edu 